Marino Badiale · Vieri Benci · Sergio Rolando

\title{
A nonlinear elliptic equation with singular potential and applications to nonlinear field equations
}

Received July 6, 2005 and in revised form January 31, 2006

Abstract. We prove the existence of cylindrical solutions to the semilinear elliptic problem

$$
-\Delta u+\frac{u}{|y|^{2}}=f(u), \quad u \in H^{1}\left(\mathbb{R}^{N}\right), \quad u \geq 0,
$$

where $(y, z) \in \mathbb{R}^{k} \times \mathbb{R}^{N-k}, N>k \geq 2$ and $f$ has a double-power behaviour, subcritical at infinity and supercritical near the origin.

This result also implies the existence of solitary waves with nonvanishing angular momentum for nonlinear Schrödinger and Klein-Gordon equations.

\section{Introduction}

This paper is mainly concerned with the existence of nontrivial cylindrical solutions for the semilinear singular elliptic problem

$$
-\Delta u+\frac{u}{|y|^{2}}=f(u), \quad u \in H^{1}\left(\mathbb{R}^{N}\right), \quad u \geq 0
$$

where $(y, z) \in \mathbb{R}^{k} \times \mathbb{R}^{N-k}, N>k \geq 2$ and $f \in C(\mathbb{R} ; \mathbb{R})$ is such that $f(0)=0$. Other working assumptions on $f$ will be made in Section 2 , in particular we will ask $f$ to satisfy a double-power growth condition, supercritical near the origin and subcritical at infinity (more precisely, see assumptions $\left(\mathbf{f}_{1}\right)-\left(\mathbf{f}_{4}\right)$ ).

Problems like (1) arise in the search for finite-energy stationary solutions to nonlinear evolution equations of the Schrödinger or Klein-Gordon type. The finiteness of energy makes the solutions physically meaningful and it is strictly related to the finiteness of the

Research of M. Badiale and S. Rolando supported by MIUR, project "Variational Methods and Nonlinear Differential Equations".

Research of V. Benci supported by MIUR, project "Metodi variazionali e topologici nello studio di fenomeni non lineari”.

M. Badiale, S. Rolando: Dipartimento di Matematica, Università di Torino, Via Carlo Alberto 10, 10123 Torino, Italy; e-mail: marino.badiale@unito.it, sergio.rolando@unito.it

V. Benci: Dipartimento di Matematica Applicata “U. Dini”, Università di Pisa, Via Bonanno 25/b, 56126 Pisa, Italy; e-mail: benci@dma.unipi.it 
$L^{2}$ norm. Looking for stationary waves, one is led to equations of the form

$$
-\Delta u+V(x) u=f(u) \quad \text { in } \mathbb{R}^{3}
$$

for which a large number of existence results have been established under very different assumptions on the potential $V$ and the nonlinearity $f$ (see for instance the references in [3], [19]). However, it is mostly assumed that $V$ is bounded away from zero, so that an $H^{1}$ variational approach is permitted (as regards the case $V=0$, see for example [12], [29] and the references therein). Among the papers which deal instead with potentials satisfying $V \neq 0$ and $\liminf _{|x| \rightarrow \infty} V(x)=0$, let us cite the following recent works (see [31] for further references). In [1] the existence of a positive finite-energy solution is proved in the case of smooth potentials and nonlinearities (not necessarily radial) having the form $V(x) \sim\left(1+|x|^{a}\right)^{-1}$ and $f(x, u) \sim\left(1+|x|^{b}\right)^{-1}|u|^{p}$ with $0<a<2$, $b>0$ and $\max \{1,5-4 b / a\}<p<5$. The presence of singularities in equation (2) is allowed in [11], where it is required that $V \in L^{3 / 2} \cap L^{r}\left(\mathbb{R}^{3}\right)$ for some $r>3 / 2$ with $\|V\|_{L^{3 / 2}}$ suitably small and $f$ satisfies assumptions similar to $\left(\mathbf{f}_{1}\right)$ and $\left(\mathbf{f}_{3}\right)$. In [23] the problem of positive solutions is studied for critical nonlinearity and potentials featuring multiple inverse-square singularities. Existence (and nonexistence) results for radial potentials $V(x) \geq A|x|^{-\alpha}$ satisfying mild integrability assumptions can be found in [4], where both subcritical and supercritical nonlinearities are considered. The case of radial potentials and double-power nonlinearities is treated in [5]. However, no results on the finiteness of energy are proved in [4], [5], [11], [23]. Theorem 3 of Section 2 below provides a nontrivial, well localized in space, finite-energy solution to equation (2) in the case of the singular cylindrical potential $V(x)=|y|^{-2}$ (see again [5] for results concerning cylindrical potentials in high dimensions). Let us point out that the technique used in [1] to bound the $L^{2}$ norm does not suit our case, for it is strictly related to the assumption $a<2$.

Though we are interested here in entire solutions of (2), it is worth observing that nonlinear elliptic equations with singular potentials have been widely investigated also on bounded domains of $\mathbb{R}^{N}$, especially in the presence of critical power nonlinearities. For instance (other references can be found in [31]), the case $V(x)=\lambda+\mu|x|^{-2}$ is studied in [24], [27], [32] (see also [18]), where the solvability of the equation is examined in connection with the sign and size of the parameters $\lambda, \mu$. In the presence of more general nonlinearities of the form $f(x, u)=u^{p}+\lambda b(x)$, inverse-square potentials $V(x)=-A|x|^{-2}$ with $0<A \leq(N-2)^{2} / 4$ are considered in [16] (case $\lambda=0$ ) and [20] (case $\lambda>0$ ), where compatibility conditions on $A, p, \lambda$ and the space dimension are exhibited in order to ensure the existence of solutions. The results of [20] are extended in [21] to a larger class of potentials and nonlinearities. This kind of investigation is related to the study of improved Hardy inequalities (see for example [17] and [30]).

As another remarkable application of problem (1), we derive the existence of finiteenergy waves with nonvanishing angular momentum for evolution field equations. Let us briefly introduce the matter, which will be the topic of Section 7 Consider for instance the nonlinear Schrödinger equation

$$
i \frac{\partial \psi}{\partial t}=-\Delta \psi+\omega \psi-f(|\psi|)|\psi|^{-1} \psi, \quad(x, t) \in \mathbb{R}^{3} \times \mathbb{R},
$$


where $\omega \in \mathbb{R}$. It is well known that equation (3) has stationary solutions with nonvanishing angular momentum of the form

$$
\psi(x, t)=\varphi(x) e^{-i \Omega t}, \quad \varphi(x) \in \mathbb{C}, \Omega \in \mathbb{R},
$$

with $\Omega<\omega$. Here we will show that also the limit case $\Omega=\omega$ can be achieved. In this case, equation (3) is equivalent to

$$
-\Delta \varphi=f(|\varphi|)|\varphi|^{-1} \varphi \quad \text { in } \mathbb{R}^{3} .
$$

If we make the ansatz $\varphi(x)=u(x) e^{i \vartheta(y)}, u(x) \geq 0$, where $\vartheta(y)$ gives the angle of the cylindrical coordinates in $\mathbb{R}^{3}$, equation $(5)$ also turns out to be equivalent to equation (1), provided that $u(y, z)=u(|y|, z)$. Hence, by studying equation (1), we obtain a nontrivial entire classical solution $\varphi \in H^{1}\left(\mathbb{R}^{3}\right)$ to equation (5) (Theorem 27). Then by means of 4 ), with $\Omega=\omega$, one sees that equation $(3)$ admits finite-energy standing (and also travelling) waves which are well localized in space and bear a nonvanishing angular momentum, given by $\mathbf{M}(\psi)=-\left(0,0,\|u\|_{L^{2}\left(\mathbb{R}^{3}\right)}^{2}\right)$ (Theorem 28 and Remark 29$)$. The same argument applies to nonlinear Klein-Gordon equations (Remark 30). To our knowledge, the only papers concerning wave solutions with nonvanishing angular momentum are [13] and [19] (see also [8]), in which the equation

$$
i \frac{\partial \psi}{\partial t}=-\Delta \psi+V(x) \psi-|\psi|^{p-2} \psi, \quad(x, t) \in \mathbb{R}^{3} \times \mathbb{R},
$$

is studied. In particular, [13] deals with the case $V=0$ and $2<p<6$, whereas in [19] it is assumed that $2<p<1+\sqrt{5}$ and $V \in C^{1} \cap L^{\infty}\left(\mathbb{R}^{3}\right)$ is bounded away from zero and has some properties related to a required cylindrical symmetry (which allows one to speak about conserved angular momentum).

We conclude this introductory section by discussing the main features of problem (1) and by sketching our approach.

Since the equation in (1) has a variational structure, its solutions can be recovered as critical points of a functional $I$ defined on a suitable function space. However the presence of a potential vanishing as $|y| \rightarrow \infty$ prevents the use of $H^{1}$ variational theory. To overcome this difficulty, we set the equation in the exact space on which the Euler functional of the linear part of the equation is well defined, namely the weighted Hilbert space, say $X\left(\mathbb{R}^{N}\right)$, consisting of the functions having finite $L^{2}$ gradient norm and such that $\int_{\mathbb{R}^{N}}|y|^{-2} u^{2} d x<\infty$. By the results of [6], the latter condition is redundant if $k>2$ (see Remark 7), whereas it is necessary in the case $k=2$. Since our assumptions ensure that $|f(t)| \leq$ const $\cdot|t|^{2^{*-1}}$ (see $\left(\mathbf{f}_{1}^{\prime}\right)$ ), also the functional related to the nonlinearity makes sense on $X\left(\mathbb{R}^{N}\right)$.

Our solution will be obtained as a critical point for the functional $I$, restricted to the closed subspace of the fixed points for the action on $X\left(\mathbb{R}^{N}\right)$ of the orthogonal group of $\mathbb{R}^{k}$. Assuming standard hypotheses on $f$ (see $\left(\mathbf{f}_{3}\right)$ and $\left(\mathbf{f}_{4}\right)$ ), the starting point is the bounded Palais-Smale sequence provided by the "mountain-pass" geometry exhibited by $I$ (on the subject see the celebrated paper [2], or some recent books such as [26], [28], [34]). Since we cannot guarantee the fulfilment of the Palais-Smale condition, such a sequence is 
studied by means of the concentration-compactness principle, in a version due to S. Solimini [33]. In ruling out the possibility of a vanishing weak limit, we exploit the growth conditions assumed on $f$, namely

$$
f(t)=O\left(|t|^{p-1}\right) \quad \text { as } t \rightarrow \infty \quad \text { and } \quad f(t)=O\left(|t|^{q-1}\right) \quad \text { as } t \rightarrow 0
$$

where $p$ and $q$ are subcritical and supercritical exponents respectively (see hypothesis $\left.\left(\mathbf{f}_{1}\right)\right)$. Probably these conditions were first considered in [14]. More recently, they have also been used in [5], [9], [10], [11], [12], [29]. The nonnegativity of the critical points of $I$ is ensured by standard hypotheses (see $\left(\mathbf{f}_{2}\right)$ ).

Since we are interested in solutions which belong to $H^{1}$, while our critical points are just in $X\left(\mathbb{R}^{N}\right)$, some more work is needed. First, thanks to a result due to E. Egnell [22], we can provide an estimate of the decay rate of the solutions. Then we exploit this asymptotic behaviour to develop a comparison argument in order to bound the $L^{2}$ norm on exterior domains. As a by-product, an improved decay estimate is also obtained. The main result of this paper is Theorem 3

Some rather standard or technical computations will be omitted in this paper; for all of them the reader is referred to [31]. Hereafter we will use the following notations.

- We shall always write $x=(y, z) \in \mathbb{R}^{k} \times \mathbb{R}^{N-k}$ with $k, N \in \mathbb{N}$ such that $N>k \geq 2$.

- $\mathbb{N}$ is the set of natural numbers, including 0 .

- For any $a \in \mathbb{R}$ we set $a_{+}:=(|a|+a) / 2$ and $a_{-}:=(|a|-a) / 2$, so that $a=a_{+}-a_{-}$ with $a_{+}, a_{-} \geq 0$.

- The open ball $B_{R}\left(\xi_{0}\right):=\left\{\xi \in \mathbb{R}^{d}:\left|\xi-\xi_{0}\right|<R\right\}$ will be simply denoted by $B_{R}$ whenever $\xi_{0}=0$. We will also write $B_{R}^{(d)}$ to make the dimension $d$ explicit. $\bar{B}_{R}$ stands for the closure of $B_{R}$.

- $|A|$ and $\chi_{A}$ respectively denote the Lebesgue measure and the characteristic function of any measurable set $A \subseteq \mathbb{R}^{d}$.

- $O(d)$ is the orthogonal group of $\mathbb{R}^{d}$.

- $L^{r}\left(\mathbb{R}^{d}\right)$ and $H^{1}\left(\mathbb{R}^{d}\right)$ are the usual Lebesgue and Sobolev spaces. Sometimes, if no misunderstanding is possible, we will briefly write $L^{r}$ and $H^{1}$.

- $r^{\prime}=r /(r-1)$ is the Hölder-conjugate exponent of $r$, so that $L^{r^{\prime}}$ is the dual of $L^{r}$.

- $2^{*}=2 N /(N-2)$ is the critical exponent for Sobolev embedding in dimension $N \geq 3$.

- $C$ will stand for any positive constant, which may change from line to line.

\section{Statement of the main results}

For $N>k \geq 2$, we consider the semilinear elliptic equation

$$
-\Delta u+\frac{u}{|y|^{2}}=f(u), \quad(y, z) \in \mathbb{R}^{k} \times \mathbb{R}^{N-k},
$$

with $u: \mathbb{R}^{N} \rightarrow \mathbb{R}$ and $f \in C(\mathbb{R} ; \mathbb{R})$ satisfying the following assumptions:

(f $\left.\mathbf{f}_{1}\right)$ there exist $M, p, q>0$ with $2<p<2^{*}<q$ such that

$$
|t| \geq 1 \Rightarrow|f(t)| \leq M|t|^{p-1} \quad \text { and } \quad|t| \leq 1 \Rightarrow|f(t)| \leq M|t|^{q-1} ;
$$


(f) $t \leq 0 \Rightarrow f(t) \geq 0$;

$\left(\mathbf{f}_{3}\right)$ there exists $\sigma>2$ such that

$$
F(t) \leq \frac{1}{\sigma} f(t) t \quad \text { for all } t \in \mathbb{R}
$$

where $F(t):=\int_{0}^{t} f(s) d s$ for any $t \in \mathbb{R} ;$

$\left(\mathbf{f}_{4}\right)$ there exists $t_{*} \in \mathbb{R}$ such that $F\left(t_{*}\right)>0$.

Example 1. It is easy to see that the assumptions $\left(\mathbf{f}_{1}\right)-\left(\mathbf{f}_{4}\right)$ are satisfied by

$$
f(t)=\left\{\begin{array}{ll}
0 & \text { for } t \leq 0 \\
\frac{t^{q-1}}{1+t^{q-p}} & \text { for } t>0
\end{array} \quad \text { with } 2<p<2^{*}<q .\right.
$$

Let us observe that from assumption $\left(\mathbf{f}_{1}\right)$ one readily deduces that

$\left(\mathbf{f}_{1}^{\prime}\right)$ there exists $M>0$ such that $|f(t)| \leq M|t|^{2^{*}-1}$ for all $t \in \mathbb{R}$,

which in turn implies that

(F) there exists $C>0$ such that $|F(t)| \leq C|t|^{2^{*}}$ for all $t \in \mathbb{R}$.

We will work in the linear space

$$
X\left(\mathbb{R}^{N}\right):=\left\{u \in D^{1,2}\left(\mathbb{R}^{N}\right): \int_{\mathbb{R}^{N}} \frac{u^{2}}{|y|^{2}} d x<\infty\right\}
$$

whose properties of interest to us are studied in Section 3 . By $D^{1,2}\left(\mathbb{R}^{N}\right)$ we mean the completion of $C_{c}^{\infty}\left(\mathbb{R}^{N}\right)$ with respect to the norm $\|u\|_{D^{1,2}}=\|\nabla u\|_{L^{2}\left(\mathbb{R}^{N}\right)}$. Observe that $X\left(\mathbb{R}^{N}\right)=D^{1,2}\left(\mathbb{R}^{N}\right)$ if $k>2$ (see Remark 7 ). We will also consider the linear subspace

$$
X_{S}\left(\mathbb{R}^{N}\right):=\left\{u \in X\left(\mathbb{R}^{N}\right): u(y, z)=u(|y|, z)\right\}
$$

where, with a slight abuse of notation, writing $u(y, z)=u(|y|, z)$ we naturally mean $u(y, z)=u(g y, z)$ for any rotation $g: \mathbb{R}^{k} \rightarrow \mathbb{R}^{k}$ and almost every $(y, z) \in \mathbb{R}^{N}$.

Our main result is Theorem 3 , which states the existence of weak solutions to equation (6) in the sense of the following definition.

Definition 2. Let $f \in C(\mathbb{R} ; \mathbb{R})$ satisfy $\left(\mathbf{f}_{1}^{\prime}\right)$. We say that $u \in X\left(\mathbb{R}^{N}\right)$ is a weak solution of equation (6) if

$$
\int_{\mathbb{R}^{N}} \nabla u \cdot \nabla \varphi d x+\int_{\mathbb{R}^{N}} \frac{u \varphi}{|y|^{2}} d x=\int_{\mathbb{R}^{N}} f(u) \varphi d x \quad \text { for all } \varphi \in H^{1}+X\left(\mathbb{R}^{N}\right) .
$$

Theorem 3. Assume that $f \in C(\mathbb{R} ; \mathbb{R})$ satisfies hypotheses $\left(\mathbf{f}_{1}\right)-\left(\mathbf{f}_{4}\right)$. Then equation 6 has a nontrivial weak solution $u \in H^{1} \cap L^{\infty} \cap X_{s}\left(\mathbb{R}^{N}\right)$ such that $u \geq 0$ and

$$
\limsup _{|x| \rightarrow \infty}|x|^{v} u(x)<\infty \quad \text { for any } v<\frac{N-2+\sqrt{(N-2)^{2}+4}}{2} .
$$


Theorem 3 is a consequence of Propositions 6 and 5 below together with the following existence result.

Theorem 4. Assume that $f \in C(\mathbb{R} ; \mathbb{R})$ satisfies hypotheses $\left(\mathbf{f}_{1}\right)-\left(\mathbf{f}_{4}\right)$. Then there exists a nontrivial $u \in X_{S}\left(\mathbb{R}^{N}\right)$, $u \geq 0$, such that

$$
\int_{\mathbb{R}^{N}} \nabla u \cdot \nabla \varphi d x+\int_{\mathbb{R}^{N}} \frac{u \varphi}{|y|^{2}} d x=\int_{\mathbb{R}^{N}} f(u) \varphi d x \quad \text { for all } \varphi \in X\left(\mathbb{R}^{N}\right) .
$$

Theorem 4 will be proved in Section 4 by variational methods. The natural space related to the growth conditions $\left(\mathbf{f}_{1}\right)$ is $L^{p}\left(\mathbb{R}^{N}\right)+L^{q}\left(\mathbb{R}^{N}\right)$ (see [9], [10], [15] and [29]). In spite of that, it will not be involved in our functional setting, since many of our computations only require condition $\left(\mathbf{f}_{1}^{\prime}\right)$. However, assumption $\left(\mathbf{f}_{1}\right)$ is crucial for our existence result and it will be used directly in the proof of Lemmas 24 and 25 . As usual, hypothesis $\left(\mathbf{f}_{3}\right)$ is used to prove the boundedness of the Palais-Smale sequences of the Euler functional $I$ related to equation (6) and defined on $X\left(\mathbb{R}^{N}\right)$. Such a sequence is provided by the "mountain-pass" geometry of $I$, which is granted by assumption $\left(\mathbf{f}_{4}\right)$, and will be studied by means of the concentration-compactness principle, in a version due to Solimini [33]. Finally, condition $\left(\mathbf{f}_{2}\right)$ is assumed to yield the nonnegativity of the critical points of $I$.

Once Theorem 4 is proved, to get Theorem 3 we need to study the asymptotic and summability properties of the weak solution $u \geq 0$ obtained; in particular we have to show that $u$ actually belongs to $H^{1}$. Moreover, we have to ensure that equation 8 is satisfied by any test function $\varphi \in H^{1}$. These are the aims of the following propositions.

Proposition 5. Let $f \in C(\mathbb{R} ; \mathbb{R})$ satisfy $\left(\mathbf{f}_{1}^{\prime}\right)$. If $u \in X\left(\mathbb{R}^{N}\right)$ is such that $u \geq 0$ and 8 holds, then

$$
\int_{\mathbb{R}^{N}} \nabla u \cdot \nabla \varphi d x+\int_{\mathbb{R}^{N}} \frac{u \varphi}{|y|^{2}} d x=\int_{\mathbb{R}^{N}} f(u) \varphi d x \quad \text { for all } \varphi \in H^{1}\left(\mathbb{R}^{N}\right) .
$$

Proposition 6. Let $f \in C(\mathbb{R} ; \mathbb{R})$ satisfy $\left(\mathbf{f}_{1}^{\prime}\right)$. Then any weak solution $u \geq 0$ of equation (6) is bounded, belongs to $L^{2}\left(\mathbb{R}^{N}\right)$ and satisfies (7).

Proposition 5, which is trivial for $k>2$ (Remark 7), is an extendibility result if $k=2$, and it will be proved in Section 5 .

In Proposition 6 we study properties of nonnegative weak solutions. The proof, which is given in Section 6 relies on a comparison argument and takes advantage of asymptotic results due to Egnell [22].

As announced in the introduction, Theorem 3 yields some other existence result, regarding the case $N=3$. Needing some preliminary discussion, they will be stated and proved in Section 7

\section{Functional setting}

\subsection{A weighted Sobolev space}

We will work in the Hilbert space

$$
D^{1,2}:=D^{1,2}\left(\mathbb{R}^{N}\right)=\left\{u \in L^{2^{*}}\left(\mathbb{R}^{N}\right): \nabla u \in L^{2}\left(\mathbb{R}^{N}\right)\right\}
$$


endowed with the norm $\|u\|_{D^{1,2}}=\|\nabla u\|_{L^{2}}$. Let us also recall that, for any given open subset $\Omega \subseteq \mathbb{R}^{N}, D_{0}^{1,2}(\Omega)$ denotes the closure of $C_{c}^{\infty}(\Omega)$ in $D^{1,2}(\Omega)=\left\{u \in L^{2^{*}}(\Omega)\right.$ : $\left.\nabla u \in L^{2}(\Omega)\right\}$ with respect to the norm $\|u\|_{D^{1,2}(\Omega)}=\|u\|_{L^{2^{*}}(\Omega)}+\|\nabla u\|_{L^{2}(\Omega)}$. We define

$$
X:=X\left(\mathbb{R}^{N}\right)=\left\{u \in D^{1,2}: \int_{\mathbb{R}^{N}} \frac{u^{2}}{|y|^{2}} d x<\infty\right\},
$$

which is a Hilbert space with respect to the norm

$$
\|u\|^{2}:=\int_{\mathbb{R}^{N}}|\nabla u|^{2} d x+\int_{\mathbb{R}^{N}} \frac{u^{2}}{|y|^{2}} d x
$$

induced by the scalar product

$$
(u \mid v):=\int_{\mathbb{R}^{N}} \nabla u \cdot \nabla v d x+\int_{\mathbb{R}^{N}} \frac{u v}{|y|^{2}} d x .
$$

Clearly

$$
X_{s}:=X_{s}\left(\mathbb{R}^{N}\right)=\{u \in X: u(y, z)=u(|y|, z)\}
$$

is a closed subspace of $X$. Let us point out that weak convergence in $X$ (or $X_{S}$ ) implies pointwise convergence (up to a subsequence and almost everywhere).

Remark 7. If $k>2$ then, by the Sobolev-Hardy inequalities [6], one has

$$
\forall u \in D^{1,2}\left(\mathbb{R}^{N}\right) \quad \int_{\mathbb{R}^{N}} \frac{u^{2}}{|y|^{2}} d x \leq\left(\frac{2}{k-2}\right)^{2} \int_{\mathbb{R}^{N}}|\nabla u|^{2} d x .
$$

Hence $X=D^{1,2}\left(\mathbb{R}^{N}\right)$ and the norms $\|\cdot\|$ and $\|\cdot\|_{D^{1,2}}$ are equivalent.

Remark 8. If $\tilde{z}_{0}=\left(0, z_{0}\right) \in \mathbb{R}^{N}, \lambda>0, g \in O(k)$ and $u \in X$, then

$$
\left\|u\left(\lambda^{-1} \cdot+\tilde{z}_{0}\right)\right\|=\lambda^{(N-2) / 2}\|u\| \quad \text { and } \quad(u \mid v(g \cdot, \cdot))=\left(u\left(g^{-1} \cdot, \cdot\right) \mid v\right) .
$$

Of course $X_{s}=\{u \in X: u(g \cdot, \cdot)=u$ for all $g \in O(k)\}$.

For later use, we recall here a result due to S. Solimini [33]. First we have to introduce a group of rescaling operators, of which we also give the basic properties that will be useful in the following.

Definition 9. Fix $\lambda>0$ and $\xi \in \mathbb{R}^{N}$. For any $u \in L^{r}\left(\mathbb{R}^{N}\right)$ with $1<r<\infty$ we define

$$
T(\lambda, \xi) u:=\lambda^{-(N-2) / 2} u\left(\lambda^{-1} \cdot+\xi\right) .
$$

Clearly $T(\lambda, \xi) u \in L^{r}$ for all $u \in L^{r}$ and in particular $T(\lambda, \xi) u \in D^{1,2}$ if $u \in D^{1,2}$. Moreover, by direct computations, it is easy to see that the linear operators $u \in L^{2^{*}} \mapsto$ $T(\lambda, \xi) u \in L^{2^{*}}$ and $u \in D^{1,2} \mapsto T(\lambda, \xi) u \in D^{1,2}$ are isometric. Notice that

$$
T(\lambda, \xi)^{-1}=T(1 / \lambda,-\lambda \xi) \quad \text { and } \quad T(\lambda, \xi) T(\mu, \eta)=T(\lambda \mu, \xi / \mu+\eta) .
$$


Remark 10. For any $\tilde{z}_{0}=\left(0, z_{0}\right) \in \mathbb{R}^{N}$ and $\lambda>0$, from Remark 8 we readily deduce that the linear operator $u \in X \mapsto T\left(\lambda, \tilde{z}_{0}\right) u \in X$ is an isometry. Clearly $T\left(\lambda, \tilde{z}_{0}\right) u \in X_{s}$ if $u \in X_{s}$.

Proposition 11. Let $1<r<\infty$ and assume that $\left\{\lambda_{n}\right\} \subset(0, \infty)$ and $\left\{\xi_{n}\right\} \subset \mathbb{R}^{N}$ are such that $\lambda_{n} \rightarrow \lambda \neq 0$ and $\xi_{n} \rightarrow \xi$. Then

$$
T\left(\lambda_{n}, \xi_{n}\right) u_{n} \rightarrow T(\lambda, \xi) u \quad \text { in } L^{r}\left(\mathbb{R}^{N}\right)
$$

if $u_{n} \rightarrow u$ in $L^{r}\left(\mathbb{R}^{N}\right)$.

Proof. Let $T_{n}:=T\left(\lambda_{n}, \xi_{n}\right)$ and $T:=T(\lambda, \xi)$. Applying the Lebesgue dominated convergence theorem, it is easy to check that

$$
T_{n}^{-1} \varphi \rightarrow T^{-1} \varphi \quad \text { in } L^{r^{\prime}}
$$

for any $\varphi \in C_{c}^{\infty}\left(\mathbb{R}^{N}\right)$. Arguing by density, we can conclude that the convergence actually holds for any $\varphi \in L^{r^{\prime}}$. As a consequence, by obvious changes of variables, we get

$$
\int_{\mathbb{R}^{N}}\left(T_{n} u_{n}\right) \varphi d x=\lambda_{n}^{2} \int_{\mathbb{R}^{N}} u_{n}\left(T_{n}^{-1} \varphi\right) d x \rightarrow \lambda^{2} \int_{\mathbb{R}^{N}} u\left(T^{-1} \varphi\right) d x=\int_{\mathbb{R}^{N}}(T u) \varphi d x
$$

for any $\varphi \in L^{r^{\prime}}$.

Remark 12. Let $\left\{\lambda_{n}\right\} \subset(0, \infty)$ and $\left\{\tilde{z}_{n}\right\} \subset\{0\} \times \mathbb{R}^{N-k}$ be such that $\lambda_{n} \rightarrow \lambda \neq 0$ and $\tilde{z}_{n} \rightarrow \tilde{z}$. By Proposition 11 and Remark 10 it is easy to see that if $u_{n} \rightarrow u$ in $X$ (or $X_{s}$ ) then, up to a subsequence, $T\left(\lambda_{n}, \tilde{z}_{n}\right) u_{n} \rightarrow T(\lambda, \tilde{z}) u$ in $X$ (or $X_{s}$ ).

The main result we will exploit about these rescalings is the following, due to S. Solimini. It is a version of the concentration-compactness principle.

Theorem 13 ([33]). If $\left\{u_{n}\right\} \subset D^{1,2}\left(\mathbb{R}^{N}\right)$ is bounded, then, up to a subsequence, either $u_{n} \rightarrow 0$ in $L^{2^{*}}\left(\mathbb{R}^{N}\right)$ or there exist $\left\{\lambda_{n}\right\} \subset(0, \infty)$ and $\left\{\xi_{n}\right\} \subset \mathbb{R}^{N}$ such that $T\left(\lambda_{n}, \xi_{n}\right) u_{n}$ $\rightarrow u$ in $L^{2^{*}}\left(\mathbb{R}^{N}\right)$ and $u \neq 0$.

\subsection{The Euler functional}

Assume that $f \in C(\mathbb{R} ; \mathbb{R})$ satisfies hypothesis $\left(\mathbf{f}_{1}^{\prime}\right)$ and set

$$
I(u):=\frac{1}{2}\|u\|^{2}-\int_{\mathbb{R}^{N}} F(u) d x .
$$

Thanks to condition $\left(\mathbf{f}_{1}^{\prime}\right)$, by standard computations one proves that $I: X \rightarrow \mathbb{R}$ is of class $C^{1}$ with Fréchet derivative $I^{\prime}(u)$ at any $u \in X$ given by

$$
\forall h \in X \quad I^{\prime}(u) h=(u \mid h)-\int_{\mathbb{R}^{N}} f(u) h d x .
$$


Proposition 14. For any $h \in X\left(\mathbb{R}^{N}\right)$ the mapping $I^{\prime}(\cdot) h: X\left(\mathbb{R}^{N}\right) \rightarrow \mathbb{R}$ is sequentially weakly continuous.

Proof. Of course we need only consider the nonlinear term of the mapping. So we fix $h \in D^{1,2}$ and show the sequential weak continuity on $D^{1,2}$ of the mapping $u \mapsto \int_{\mathbb{R}^{N}} f(u) h d x$. Accordingly, assuming $u_{n} \rightarrow u$ in $D^{1,2}$, we need to show that $\int_{\mathbb{R}^{N}} f\left(u_{n}\right) h d x \rightarrow \int_{\mathbb{R}^{N}} f(u) h d x$. With a view to arguing by density, let $\varphi \in C_{c}^{\infty}\left(\mathbb{R}^{N}\right)$ and let $R>0$ be such that $\operatorname{supp} \varphi \subset B_{R}$. From the compactness of the embedding $D^{1,2}\left(\mathbb{R}^{N}\right) \hookrightarrow L^{2^{*}-1}\left(B_{R}\right)$ we deduce that $u_{n} \rightarrow u$ in $L^{2^{*}-1}\left(B_{R}\right)$. On the other hand, condition $\left(\mathbf{f}_{1}^{\prime}\right)$ ensures the continuity of the Nemytskiu operator $f: L^{2^{*}-1}\left(B_{R}\right) \rightarrow L^{1}\left(B_{R}\right)$. Hence $\int_{\mathbb{R}^{N}}\left|f\left(u_{n}\right)-f(u)\right||\varphi| d x \rightarrow 0$. Now, by $\left(\mathbf{f}_{1}^{\prime}\right)$ and the boundedness of $\left\{u_{n}\right\}$ in $L^{2^{*}}$, one has

$$
\int_{\mathbb{R}^{N}}\left|f\left(u_{n}\right)-f(u)\right||h| d x \leq C\|h-\varphi\|_{L^{2^{*}}}+\int_{\mathbb{R}^{N}}\left|f\left(u_{n}\right)-f(u)\right||\varphi| d x .
$$

The density of $C_{c}^{\infty}\left(\mathbb{R}^{N}\right)$ in $D^{1,2}\left(\mathbb{R}^{N}\right)$ allows us to conclude.

Proposition 15. (i) For all $u \in X$ and $\lambda>0$ we have

$$
I\left(u\left(\lambda^{-1} \cdot\right)\right)=\frac{\lambda^{N-2}}{2}\|u\|^{2}-\lambda^{N} \int_{\mathbb{R}^{N}} F(u) d x .
$$

(ii) If $u \in X_{s}$ then for all $h \in X$ and $g \in O(k)$ we have

$$
I^{\prime}(u) h(g \cdot, \cdot)=I^{\prime}(u) h .
$$

Proof. By suitable changes of variables, it is a straightforward computation.

Remark 16. Define the gradient $\nabla I(u)$ of $I$ at $u \in X$ by $\nabla I(u) \in X$ and $(\nabla I(u) \mid h)=$ $I^{\prime}(u) h$ for all $h \in X$. By 100 and Proposition 15 (ii) it is easy to see that $u \in X_{s}$ implies $\nabla I(u) \in X_{s}$, so that $(\nabla I(u) \mid h)=0$ for all $h \in X_{s}$ means $\nabla I(u)=0$.

Lemma 17. If $\left(\mathbf{f}_{4}\right)$ holds, then there exists $u \in X_{s}$ such that $\int_{\mathbb{R}^{N}} F(u) d x>0$.

Proof. For $R \geq 3$, let $\phi_{R} \in C_{c}^{\infty}(\mathbb{R} ; \mathbb{R})$ be such that $0 \leq \phi_{R} \leq 1$ with $\phi_{R}(t)=0$ for $t \leq 1$ or $t \geq R+1$ and $\phi_{R}(t)=1$ for $2 \leq t \leq R$. Setting $u_{R}(x):=\phi_{R}(|y|) \phi_{R}(|z|)$ for all $x \in \mathbb{R}^{N}$, one checks that $\int_{\mathbb{R}^{N}} F\left(t_{*} u_{R}\right) d x \geq C R^{N}+o\left(R^{N}\right)$ as $R \rightarrow \infty$.

Proposition 18. If assumptions $\left(\mathbf{f}_{3}\right)$ and $\left(\mathbf{f}_{4}\right)$ hold, then there exist $c>0$ and a bounded sequence $\left\{w_{n}\right\} \subset X_{s}$ such that

$$
I\left(w_{n}\right) \rightarrow c \quad \text { and } \quad I^{\prime}\left(w_{n}\right)_{\mid X_{s}} \rightarrow 0 \quad \text { in } X_{s}^{\prime} .
$$

Proof. $I_{\mid X_{s}} \in C^{1}\left(X_{s} ; \mathbb{R}\right)$ has a "mountain-pass" geometry. Indeed, on the one hand, we have $I(0)=0$ and

$$
\|u\|=\rho \Rightarrow I(u) \geq \frac{1}{2} \rho^{2}-C \rho^{2^{*}},
$$


as follows from the fact that (F) implies $|F(u)| \leq C|u|^{2^{*}}$ so that for all $u \in X$ one has $\left|\int_{\mathbb{R}^{N}} F(u) d x\right| \leq C\|u\|^{2^{*}}$ by the Sobolev inequality. On the other hand, for any $u \in X_{S}$ such that $\int_{\mathbb{R}^{N}} F(u) d x>0$ (see Lemma 17, from 10) and Proposition 15 (i) we get

$$
\lim _{\lambda \rightarrow \infty}\left\|u\left(\lambda^{-1} \cdot\right)\right\|=\infty \text { and } \lim _{\lambda \rightarrow \infty} I\left(u\left(\lambda^{-1} \cdot\right)\right)=-\infty
$$

with $u\left(\lambda^{-1} \cdot\right) \in X_{s}$. Hence usual minimax arguments (see for instance Section 2.3 in [34]) provide the existence of a level $c>0$ and a sequence $\left\{w_{n}\right\} \subset X_{s}$ such that $I\left(w_{n}\right) \rightarrow c$ and $I^{\prime}\left(w_{n}\right)_{\mid X_{s}} \rightarrow 0$ in $X_{s}^{\prime}$. Exploiting assumption $\left(\mathbf{f}_{3}\right)$, a standard argument shows that $\left\{w_{n}\right\}$ has to be bounded in $X_{s}$.

\section{The existence result}

This section is devoted to the proof of Theorem 4, which will be achieved through several lemmata. Assume that $f \in C(\mathbb{R} ; \mathbb{R})$ satisfies hypotheses $\left(\mathbf{f}_{1}\right)-\left(\mathbf{f}_{4}\right)$. As usual, we take $N>k \geq 2$ and write $x=(y, z) \in \mathbb{R}^{k} \times \mathbb{R}^{N-k}$. Since we will very often pass from a sequence to a subsequence, for simplicity we shall maintain the same indices.

From (12) and (9) one clearly sees that any critical point of $I: X \rightarrow \mathbb{R}$ satisfies (8). On the other hand, Remark 16 shows that $X_{S}$ is a natural constraint for finding critical points of $I$. Hence, in order to prove Theorem 4, we look for nonzero critical points of $I_{\mid X_{s}}$.

The starting point is the bounded Palais-Smale sequence $\left\{w_{n}\right\} \subset X_{s}$ provided by Proposition 18, which, we recall, is such that

$$
I\left(w_{n}\right) \rightarrow c>0 \quad \text { and } \quad I^{\prime}\left(w_{n}\right) \rightarrow 0 \quad \text { in } X_{s}^{\prime} .
$$

Since $\left\{w_{n}\right\}$ is bounded in $D^{1,2}$, it must satisfy one of the alternatives allowed by Theorem 13 . We now show that the first one can easily be ruled out.

Lemma 19. The sequences $\left\{w_{n}\right\}$ does not converge to 0 in $L^{2^{*}}\left(\mathbb{R}^{N}\right)$.

Proof. Assume that $w_{n} \rightarrow 0$ in $L^{2^{*}}$. From $\left(\mathbf{f}_{1}^{\prime}\right)$ and $(\mathbf{F})$ we derive $\left|f\left(w_{n}\right) w_{n}\right| \leq C\left|w_{n}\right|^{2^{*}}$ and $\left|F\left(w_{n}\right)\right| \leq C\left|w_{n}\right|^{2^{*}}$ almost everywhere in $\mathbb{R}^{N}$, so that

$$
\int_{\mathbb{R}^{N}} f\left(w_{n}\right) w_{n} d x \rightarrow 0 \text { and } \int_{\mathbb{R}^{N}} F\left(w_{n}\right) d x \rightarrow 0 .
$$

On the other hand, $I^{\prime}\left(w_{n}\right) w_{n} \rightarrow 0$ since $I^{\prime}\left(w_{n}\right) \rightarrow 0$ in $X_{s}^{\prime}$ and $\left\{w_{n}\right\} \subset X_{s}$ is bounded. Therefore we conclude

$$
\left\|w_{n}\right\|^{2}=I^{\prime}\left(w_{n}\right) w_{n}+\int_{\mathbb{R}^{N}} f\left(w_{n}\right) w_{n} d x \rightarrow 0
$$

and thus

$$
I\left(w_{n}\right)=\frac{1}{2}\left\|w_{n}\right\|^{2}-\int_{\mathbb{R}^{N}} F\left(w_{n}\right) d x \rightarrow 0,
$$

which contradicts $I\left(w_{n}\right) \rightarrow c>0$. 
Corollary 20. Up to a subsequence, there exist $\left\{\lambda_{n}\right\} \subset(0, \infty)$ and $\left\{x_{n}\right\} \subset \mathbb{R}^{N}$ such that

$$
T\left(\lambda_{n}, x_{n}\right) w_{n} \rightarrow \tilde{w} \quad \text { in } L^{2^{*}}\left(\mathbb{R}^{N}\right) \text { and } \tilde{w} \neq 0 .
$$

Proof. Apply Theorem 13 and use Lemma19.

Now we can easily exploit the $z$-translation invariance of equation (6) to slightly improve the result of Corollary 20 To this end, we set $x_{n}=:\left(y_{n}, z_{n}\right), \tilde{y}_{n}:=\left(y_{n}, 0\right)$ and $\tilde{z}_{n}:=$ $\left(0, z_{n}\right)$, so that $x_{n}=\tilde{y}_{n}+\tilde{z}_{n}$.

Lemma 21. Let $u_{n}:=T\left(1, \tilde{z}_{n}\right) w_{n}$. Then $\left\{u_{n}\right\} \subset X_{s}$ is bounded and such that

$$
I^{\prime}\left(u_{n}\right) \rightarrow 0 \quad \text { in } X_{s}^{\prime} \quad \text { and } T\left(\lambda_{n}, \tilde{y}_{n}\right) u_{n} \rightarrow \tilde{w} \quad \text { in } L^{2^{*}}\left(\mathbb{R}^{N}\right) .
$$

Proof. Since $\left\{w_{n}\right\} \subset X_{s}$ is bounded, from Remark 10 we deduce that $\left\{u_{n}\right\} \subset X_{s}$ is bounded. Moreover, recalling (11), we have

$$
T\left(\lambda_{n}, \tilde{y}_{n}\right) u_{n}=T\left(\lambda_{n}, \tilde{y}_{n}\right) T\left(1, \tilde{z}_{n}\right) w_{n}=T\left(\lambda_{n}, x_{n}\right) w_{n} \rightarrow \tilde{w} \quad \text { in } L^{2^{*}}\left(\mathbb{R}^{N}\right) .
$$

Finally, by easy computation, one has

$$
\begin{aligned}
I^{\prime}\left(u_{n}\right) h & =\left(w_{n}\left(\cdot+\tilde{z}_{n}\right) \mid h\right)-\int_{\mathbb{R}^{N}} f\left(w_{n}\left(\cdot+\tilde{z}_{n}\right)\right) h d x \\
& =\left(w_{n} \mid h\left(\cdot-\tilde{z}_{n}\right)\right)-\int_{\mathbb{R}^{N}} f\left(w_{n}\right) h\left(\cdot-\tilde{z}_{n}\right) d x \\
& =I^{\prime}\left(w_{n}\right) T\left(1,-\tilde{z}_{n}\right) h
\end{aligned}
$$

for all $h \in X_{s}$, so that $\left\|I^{\prime}\left(u_{n}\right)\right\|_{X_{s}^{\prime}}=\left\|I^{\prime}\left(w_{n}\right)\right\|_{X_{s}^{\prime}}$ again by Remark 10

The next point in the proof of Theorem 4 is the removal of translations from the rescalings $T\left(\lambda_{n}, \tilde{y}_{n}\right)$. This is the topic of Lemma 23 , where we will take advantage of the following elementary proposition.

Proposition 22. Let $\left\{\eta_{n}\right\} \subset \mathbb{R}^{k}$ be such that $\lim _{n \rightarrow \infty}\left|\eta_{n}\right|=\infty$ and fix $R>0$. Then for any $m \in \mathbb{N} \backslash\{0,1\}$ there exists $n_{m} \in \mathbb{N}$ such that for any $n>n_{m}$ one can find $g_{1}, \ldots, g_{m} \in O(k)$ satisfying the condition

$$
i \neq j \Rightarrow B_{R}\left(g_{i} \eta_{n}\right) \cap B_{R}\left(g_{j} \eta_{n}\right)=\emptyset .
$$

Proof. Fix $m \in \mathbb{N} \backslash\{0,1\}$ and let $\left\{\xi_{1}, \ldots, \xi_{m}\right\} \subset \mathbb{R}^{k}$ be such that $\left|\xi_{i}\right|=1$ for $i=1, \ldots, m$ and

$$
\delta_{m}:=\min \left\{\left|\xi_{i}-\xi_{j}\right|: i, j=1, \ldots, m \text { with } i \neq j\right\}>0 .
$$

For all $\rho>\rho_{m}:=2 R \delta_{m}^{-1}, i \neq j$ implies

$$
\left|\rho \xi_{i}-\rho \xi_{j}\right|=\rho\left|\xi_{i}-\xi_{j}\right|>\rho_{m} \delta_{m}=2 R
$$

and hence

$$
\inf _{\xi \in B_{R}\left(\rho \xi_{i}\right), \eta \in B_{R}\left(\rho \xi_{j}\right)}|\xi-\eta| \geq\left|\rho \xi_{i}-\rho \xi_{j}\right|-2 R>0
$$

so that $B_{R}\left(\rho \xi_{i}\right) \cap B_{R}\left(\rho \xi_{j}\right)=\emptyset$. Then, since there exists $n_{m} \in \mathbb{N}$ such that $\left|\eta_{n}\right|>\rho_{m}$ for all $n>n_{m}$, the proof is completed by taking $g_{1}, \ldots, g_{m} \in O(k)$ such that $g_{i} \eta_{n}=\left|\eta_{n}\right| \xi_{i}$ for $i=1, \ldots, m$. 
Lemma 23. Up to a subsequence, we have

$$
T\left(\lambda_{n}, 0\right) u_{n} \rightarrow \tilde{u} \text { in } X_{s} \text { and } \tilde{u} \neq 0 .
$$

Proof. Let $\tilde{T}_{n}:=T\left(\lambda_{n}, \tilde{y}_{n}\right)$ and

$$
v_{n}:=T\left(\lambda_{n}, 0\right) u_{n} .
$$

From Remark 10 we get $v_{n} \in X_{s}$ and $\left\|v_{n}\right\|=\left\|u_{n}\right\|$, so that (up to a subsequence) we can assume $v_{n} \rightarrow \tilde{u}$ in $X_{s}$. If $\tilde{u} \neq 0$ the proof is complete. We now show by contradiction that $\tilde{u}=0$ is impossible. So, assume

$$
v_{n} \rightarrow 0 \quad \text { in } X_{s} .
$$

We recall from Lemma 21 that $\tilde{T}_{n} u_{n} \rightarrow \tilde{w} \neq 0$ in $L^{2^{*}}$.

First, we deduce that

$$
\lim _{n \rightarrow \infty}\left|\lambda_{n} \tilde{y}_{n}\right|=\infty .
$$

Otherwise, up to a subsequence $\lambda_{n} \tilde{y}_{n} \rightarrow \tilde{y}_{0} \in \mathbb{R}^{k} \times\{0\}$ and

$$
T\left(1,-\lambda_{n} \tilde{y}_{n}\right) \tilde{T}_{n} u_{n} \rightarrow T\left(1,-\tilde{y}_{0}\right) \tilde{w} \quad \text { in } L^{2^{*}}
$$

by Proposition 11 But, since $T\left(1,-\lambda_{n} \tilde{y}_{n}\right) \tilde{T}_{n}=T\left(\lambda_{n}, 0\right)$, this means $v_{n} \rightarrow T\left(1,-\tilde{y}_{0}\right) \tilde{w}$ $\neq 0$ in $L^{2^{*}}$, which contradicts 13 .

Since $\tilde{w} \neq 0$, there exist $\delta>0$ and $A \subseteq \mathbb{R}^{N}$ with $|A| \neq 0$ such that either $\tilde{w}>\delta$ or $\tilde{w}<-\delta$ almost everywhere in $A$. Fixing $R>0$ such that $\left|B_{R} \cap A\right|>0$, by weak convergence we obtain

$$
\left|\int_{\mathbb{R}^{N}} \tilde{T}_{n} u_{n} \chi_{B_{R} \cap A} d x\right| \rightarrow\left|\int_{\mathbb{R}^{N}} \tilde{w} \chi_{B_{R} \cap A} d x\right| \geq \delta\left|B_{R} \cap A\right|>0 .
$$

On the other hand, $\tilde{T}_{n} u_{n}=\tilde{T}_{n} T\left(\lambda_{n}, 0\right)^{-1} v_{n}=T\left(1, \lambda_{n} \tilde{y}_{n}\right) v_{n}$ and hence

$$
\begin{aligned}
\left|\int_{\mathbb{R}^{N}} \tilde{T}_{n} u_{n} \chi_{B_{R} \cap A} d x\right| & \leq \int_{B_{R}}\left|\tilde{T}_{n} u_{n}\right| d x=\int_{B_{R}\left(\lambda_{n} \tilde{y}_{n}\right)}\left|v_{n}\right| d x \\
& \leq C\left(\int_{B_{R}\left(\lambda_{n} \tilde{y}_{n}\right)}\left|v_{n}\right|^{2^{*}} d x\right)^{1 / 2^{*}}
\end{aligned}
$$

where $C>0$ only depends on $R$ and $N$. From 15 and 16 we now deduce that

$$
\liminf _{n \rightarrow \infty} \int_{B_{R}\left(\lambda_{n} \tilde{y}_{n}\right)}\left|v_{n}\right|^{2^{*}} d x>0
$$

and hence, up to a subsequence, we can assume

$$
\inf _{n} \int_{B_{R}\left(\lambda_{n} \tilde{y}_{n}\right)}\left|v_{n}\right|^{2^{*}} d x>\varepsilon \quad \text { for some } \varepsilon>0 .
$$


This will yield a contradiction. Indeed, using (14), from Proposition 22 it readily follows that for every $m \in \mathbb{N} \backslash\{0,1\}$ there exists $n_{m} \in \mathbb{N}$ such that for any $n>n_{m}$ one can find $g_{1}, \ldots, g_{m} \in O(k)$ satisfying the condition

$$
i \neq j \Rightarrow B_{R}\left(\lambda_{n}\left(g_{i} y_{n}, 0\right)\right) \cap B_{R}\left(\lambda_{n}\left(g_{j} y_{n}, 0\right)\right)=\emptyset .
$$

As a consequence, using (17) and the fact that $v_{n} \in X_{s}$, we get

$$
\int_{\mathbb{R}^{N}}\left|v_{n}\right|^{2^{*}} d x \geq \sum_{i=1}^{m} \int_{B_{R}\left(\lambda_{n}\left(g_{i} y_{n}, 0\right)\right)}\left|v_{n}\right|^{2^{*}} d x=\sum_{i=1}^{m} \int_{B_{R}\left(\lambda_{n} \tilde{y}_{n}\right)}\left|v_{n}\right|^{2^{*}} d x>m \varepsilon
$$

for every $m \in \mathbb{N} \backslash\{0,1\}$ and $n>n_{m}$. This finally implies

$$
\int_{\mathbb{R}^{N}}\left|v_{n}\right|^{2^{*}} d x \rightarrow \infty
$$

which is a contradiction, since $\left\|v_{n}\right\|_{L^{2^{*}}}=\left\|T\left(\lambda_{n}, 0\right) u_{n}\right\|_{L^{2^{*}}}=\left\|u_{n}\right\|_{L^{2^{*}}}$ and $\left\{u_{n}\right\}$ is bounded in $L^{2^{*}}$.

In order to apply Proposition 11 and thus to conclude that $\left\{u_{n}\right\}$ has a nonzero weak limit in $X_{s}$, we need to check that the dilation parameters $\left\{\lambda_{n}\right\}$ are actually well-behaved. This is the content of the next two lemmata.

Lemma 24. There exists $c_{1}>0$ such that $\lambda_{n} \geq c_{1}$ for all $n$, i.e. $\inf _{n} \lambda_{n}>0$.

Proof. Arguing by contradiction, up to a subsequence we assume $\lambda_{n} \rightarrow 0$ and set $T_{n}:=$ $T\left(\lambda_{n}, 0\right)$. Recall from Lemma 23 that $T_{n} u_{n} \rightarrow \tilde{u} \neq 0$ in $X_{s}$, so that (up to a subsequence) we can also assume that $T_{n} u_{n} \rightarrow \tilde{u}$ almost everywhere in $\mathbb{R}^{N}$. From Remark 10 we get $T_{n}^{-1} \tilde{u} \in X_{s}$ and $\left\|T_{n}^{-1} \tilde{u}\right\|=\|\tilde{u}\|$, so that

$$
\left|I^{\prime}\left(u_{n}\right) T_{n}^{-1} \tilde{u}\right| \leq\left\|I^{\prime}\left(u_{n}\right)\right\|_{X_{s}^{\prime}}\|\tilde{u}\| \rightarrow 0
$$

since $I^{\prime}\left(u_{n}\right) \rightarrow 0$ in $X_{s}^{\prime}$ (see Lemma 21). On the other hand, using the isometry property of $u \in X_{S} \mapsto T_{n} u \in X_{s}$ and making an obvious change of variables, we obtain

$$
\begin{aligned}
I^{\prime}\left(u_{n}\right) T_{n}^{-1} \tilde{u} & =\left(u_{n} \mid T_{n}^{-1} \tilde{u}\right)-\lambda_{n}^{(N-2) / 2} \int_{\mathbb{R}^{N}} f\left(u_{n}\right) \tilde{u}\left(\lambda_{n} \cdot\right) d x \\
& =\left(T_{n} u_{n} \mid \tilde{u}\right)-\lambda_{n}^{-(N+2) / 2} \int_{\mathbb{R}^{N}} f\left(u_{n}\left(\lambda_{n}^{-1} \cdot\right)\right) \tilde{u} d x \\
& =\left(T_{n} u_{n} \mid \tilde{u}\right)-\lambda_{n}^{-(N+2) / 2} \int_{\mathbb{R}^{N}} f\left(\lambda_{n}^{(N-2) / 2} T_{n} u_{n}\right) \tilde{u} d x .
\end{aligned}
$$

Then (18) and (19) yield

$$
a_{n}:=\left|\lambda_{n}^{-(N+2) / 2} \int_{\mathbb{R}^{N}} f\left(\lambda_{n}^{(N-2) / 2} T_{n} u_{n}\right) \tilde{u} d x\right| \rightarrow\|\tilde{u}\|^{2} \neq 0 .
$$

In order to get a contradiction, we now prove that $\lim _{n \rightarrow \infty} a_{n}=0$. 


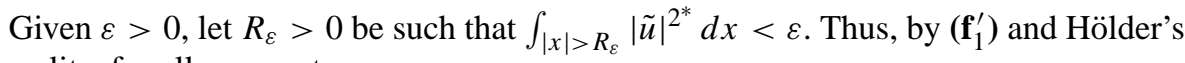
inequality, for all $n$ we get

$$
\begin{aligned}
& \left|\lambda_{n}^{-(N+2) / 2} \int_{|x|>R_{\varepsilon}} f\left(\lambda_{n}^{(N-2) / 2} T_{n} u_{n}\right) \tilde{u} d x\right| \\
& \quad \leq \lambda_{n}^{-(N+2) / 2} C \int_{|x|>R_{\varepsilon}}\left|\lambda_{n}^{(N-2) / 2} T_{n} u_{n}\right|^{2^{*}-1}|\tilde{u}| d x=C \int_{|x|>R_{\varepsilon}}\left|T_{n} u_{n}\right|^{2^{*}-1}|\tilde{u}| d x \\
& \quad \leq C\left(\int_{\mathbb{R}^{N}}\left|T_{n} u_{n}\right|^{2^{*}} d x\right)^{\left(2^{*}-1\right) / 2^{*}}\left(\int_{|x|>R_{\varepsilon}}|\tilde{u}|^{2^{*}} d x\right)^{1 / 2^{*}} \leq C_{1} \varepsilon^{1 / 2^{*}}
\end{aligned}
$$

where $C_{1}>0$ is related to $f$ and to the fact that $\left\{T_{n} u_{n}\right\}$ is bounded in $X_{s}$ and thus in $L^{2^{*}}$, but does not depend on $\varepsilon$ and $n$. Hence

$$
a_{n} \leq\left|\lambda_{n}^{-(N+2) / 2} \int_{B_{R_{\varepsilon}}} f\left(\lambda_{n}^{(N-2) / 2} T_{n} u_{n}\right) \tilde{u} d x\right|+C_{1} \varepsilon^{1 / 2^{*}} \quad \text { for all } n .
$$

With a view to studying the integral over $B_{R_{\varepsilon}}$, for any $l \in \mathbb{N}$ we define the measurable sets

$$
A_{\varepsilon, l}:=\left\{x \in B_{R_{\varepsilon}}:|\tilde{u}(x)|<l\right\}, \quad A_{\varepsilon, l}^{c}:=B_{R_{\varepsilon}} \backslash A_{\varepsilon, l},
$$

and consider, by the well known Egorov theorem on quasi-uniform convergence, a measurable set $D_{\varepsilon, l} \subseteq B_{R_{\varepsilon}}$ such that

$$
\left|B_{R_{\varepsilon}} \backslash D_{\varepsilon, l}\right|<1 / l
$$

and

$$
\forall \eta>0 \exists n_{\eta} \forall n \geq n_{\eta} \quad\left|T_{n} u_{n}-\tilde{u}\right|<\eta \quad \text { a.e. in } D_{\varepsilon, l} .
$$

Clearly $\lim _{l \rightarrow \infty}\left|A_{\varepsilon, l}^{c}\right|=0$ and $\lim _{l \rightarrow \infty}\left|A_{\varepsilon, l} \backslash\left(A_{\varepsilon, l} \cap D_{\varepsilon, l}\right)\right| \leq \lim _{l \rightarrow \infty}\left|B_{R_{\varepsilon}} \backslash D_{\varepsilon, l}\right|=0$, so that

$$
\alpha_{\varepsilon, l}:=\int_{A_{\varepsilon, l}^{c}}|\tilde{u}|^{2^{*}} d x \rightarrow 0, \quad \beta_{\varepsilon, l}:=\int_{A_{\varepsilon, l} \backslash\left(A_{\varepsilon, l} \cap D_{\varepsilon, l}\right)}|\tilde{u}|^{2^{*}} d x \rightarrow 0
$$

as $l \rightarrow \infty$ with $\varepsilon$ fixed. Then, by $\left(\mathbf{f}_{1}^{\prime}\right)$ and Hölder's inequality as before, for all $n$ we get

$$
\left|\lambda_{n}^{-(N+2) / 2} \int_{A_{\varepsilon, l}^{c}} f\left(\lambda_{n}^{(N-2) / 2} T_{n} u_{n}\right) \tilde{u} d x\right| \leq C_{1} \alpha_{\varepsilon, l}^{1 / 2^{*}}
$$

and

$$
\left|\lambda_{n}^{-(N+2) / 2} \int_{A_{\varepsilon, l} \backslash\left(A_{\varepsilon, l} \cap D_{\varepsilon, l}\right)} f\left(\lambda_{n}^{(N-2) / 2} T_{n} u_{n}\right) \tilde{u} d x\right| \leq C_{1} \beta_{\varepsilon, l}^{1 / 2^{*}}
$$

so that 20] gives

$$
a_{n} \leq\left|\lambda_{n}^{-(N+2) / 2} \int_{A_{\varepsilon, l} \cap D_{\varepsilon, l}} f\left(\lambda_{n}^{(N-2) / 2} T_{n} u_{n}\right) \tilde{u} d x\right|+C_{1}\left(\varepsilon^{1 / 2^{*}}+\alpha_{\varepsilon, l}^{1 / 2^{*}}+\beta_{\varepsilon, l}^{1 / 2^{*}}\right)
$$


for all $n$. In order to estimate the integral over $A_{\varepsilon, l} \cap D_{\varepsilon, l}$, we observe that by (21) there exists $n_{\varepsilon, l} \in \mathbb{N}$ such that

$$
\forall n \geq n_{\varepsilon, l} \quad\left|T_{n} u_{n}-\tilde{u}\right|<1 \quad \text { a.e. in } A_{\varepsilon, l} \cap D_{\varepsilon, l}
$$

and then

$$
\forall n \geq n_{\varepsilon, l} \quad\left|T_{n} u_{n}\right|<1+|\tilde{u}|<1+l \quad \text { a.e. in } A_{\varepsilon, l} \cap D_{\varepsilon, l} .
$$

As assumption ( $\mathbf{f}_{1}$ ) yields $|f(t)| \leq M|t|^{q-1}$ for all $t \in \mathbb{R}$, one obtains

$$
\begin{aligned}
&\left|\lambda_{n}^{-(N+2) / 2} \int_{A_{\varepsilon, l} \cap D_{\varepsilon, l}} f\left(\lambda_{n}^{(N-2) / 2} T_{n} u_{n}\right) \tilde{u} d x\right| \\
& \leq \lambda_{n}^{-(N+2) / 2} M \int_{A_{\varepsilon, l} \cap D_{\varepsilon, l}}\left|\lambda_{n}^{(N-2) / 2} T_{n} u_{n}\right|^{q-1}|\tilde{u}| d x \\
& \leq \lambda_{n}^{-(N+2) / 2} M \lambda_{n}^{(N-2)(q-1) / 2}(1+l)^{q-1} l\left|A_{\varepsilon, l} \cap D_{\varepsilon, l}\right| \\
&=C_{\varepsilon, l} \lambda_{n}^{(N-2)(q-1) / 2-(N+2) / 2}
\end{aligned}
$$

for all $n \geq n_{\varepsilon, l}$, where $C_{\varepsilon, l}>0$ does not depend on $n$. Together with 22, this implies

$$
a_{n} \leq C_{\varepsilon, l} \lambda_{n}^{(N-2)(q-1) / 2-(N+2) / 2}+C_{1}\left(\varepsilon^{1 / 2^{*}}+\alpha_{\varepsilon, l}^{1 / 2^{*}}+\beta_{\varepsilon, l}^{1 / 2^{*}}\right) \quad \text { for all } n \geq n_{\varepsilon, l} .
$$

Hence

$$
\limsup _{n \rightarrow \infty} a_{n} \leq C_{1}\left(\varepsilon^{1 / 2^{*}}+\alpha_{\varepsilon, l}^{1 / 2^{*}}+\beta_{\varepsilon, l}^{1 / 2^{*}}\right)
$$

since $\lambda_{n} \rightarrow 0$ and $(N-2)(q-1) / 2-(N+2) / 2=(N-2)\left(q-2^{*}\right) / 2>0$. Finally, letting first $l \rightarrow \infty$ with $\varepsilon$ fixed and then $\varepsilon \rightarrow 0$, we get $\lim _{\sup } \rightarrow \infty a_{n} \leq 0$, which means $\lim _{n \rightarrow \infty} a_{n}=0$ since $a_{n} \geq 0$. As this is a contradiction, the lemma is proved.

Lemma 25. There exists $c_{2}>0$ such that $\lambda_{n} \leq c_{2}$ for all $n$, i.e. $\sup _{n} \lambda_{n}<\infty$.

Proof. The argument is analogous to the one in the proof of Lemma 24. Up to a subsequence, we assume $\lambda_{n} \rightarrow \infty$ and $T_{n} u_{n} \rightarrow \tilde{u}$ almost everywhere in $\mathbb{R}^{N}$, where, by Lemma 23. $T_{n}:=T\left(\lambda_{n}, 0\right)$ is such that $T_{n} u_{n} \rightarrow \tilde{u} \neq 0$ in $X_{s}$. Exactly as in the proof of Lemma 24 , we deduce that

$$
a_{n}:=\left|\lambda_{n}^{-(N+2) / 2} \int_{\mathbb{R}^{N}} f\left(\lambda_{n}^{(N-2) / 2} T_{n} u_{n}\right) \tilde{u} d x\right| \rightarrow\|\tilde{u}\|^{2} \neq 0
$$

and that for any $\varepsilon>0$ there exists $R_{\varepsilon}>0$ such that

$$
a_{n} \leq\left|\lambda_{n}^{-(N+2) / 2} \int_{B_{R_{\varepsilon}}} f\left(\lambda_{n}^{(N-2) / 2} T_{n} u_{n}\right) \tilde{u} d x\right|+C_{1} \varepsilon^{1 / 2^{*}} \quad \text { for all } n
$$


where $C_{1}>0$ only depends on $f$ and $\sup _{n}\left\|T_{n} u_{n}\right\|_{L^{2^{*}}}<\infty$, not on $\varepsilon$ and $n$. Now observe that assumption ( $\left.\mathbf{f}_{1}\right)$ implies $|f(t)| \leq M|t|^{p-1}$ for every $t \in \mathbb{R}$. Hence for all $n$ we have

$$
\begin{aligned}
& \left|\lambda_{n}^{-(N+2) / 2} \int_{B_{R_{\varepsilon}}} f\left(\lambda_{n}^{(N-2) / 2} T_{n} u_{n}\right) \tilde{u} d x\right| \\
& \leq \lambda_{n}^{-(N+2) / 2} M \int_{B_{R_{\varepsilon}}}\left|\lambda_{n}^{(N-2) / 2} T_{n} u_{n}\right|^{p-1}|\tilde{u}| d x \\
& \leq \lambda_{n}^{-(N+2) / 2} M \lambda_{n}^{(N-2)(p-1) / 2} \int_{B_{R_{\varepsilon}}}\left|T_{n} u_{n}\right|^{p-1}|\tilde{u}| d x \\
& \leq M \lambda_{n}^{(N-2)(p-1) / 2-(N+2) / 2}\left(\int_{B_{R_{\varepsilon}}}\left|T_{n} u_{n}\right|^{(p-1) 2^{*} /\left(2^{*}-1\right)} d x\right)^{\left(2^{*}-1\right) / 2^{*}}\left(\int_{B_{R_{\varepsilon}}}|\tilde{u}|^{2^{*}} d x\right)^{1 / 2^{*}} \\
& \leq M \lambda_{n}^{(N-2)\left(p-2^{*}\right) / 2}\left|B_{R_{\varepsilon}}\right|^{\left(2^{*}-p\right) / 2^{*}}\left(\int_{B_{R_{\varepsilon}}}\left|T_{n} u_{n}\right|^{2^{*}} d x\right)^{(p-1) / 2^{*}}\|\tilde{u}\|_{L^{2^{*}}} \\
& \leq M \lambda_{n}^{(N-2)\left(q-2^{*}\right) / 2}\left|B_{R_{\varepsilon}}\right|^{\left(2^{*}-p\right) / 2^{*}} \sup _{n}\left\|T_{n} u_{n}\right\|_{L^{2^{*}}}^{p-1}\|\tilde{u}\|_{L^{2^{*}}} \\
& =C_{\varepsilon} \lambda_{n}^{(N-2)\left(p-2^{*}\right) / 2}
\end{aligned}
$$

where we have used the fact that $(p-1) 2^{*} /\left(2^{*}-1\right)<2^{*}$ to apply Hölder inequalities. Note that $C_{\varepsilon}>0$ does not depend on $n$, since $\left\{T_{n} u_{n}\right\}$ is bounded in $L^{2^{*}}$. Recalling $(24)$, this implies

$$
a_{n} \leq C_{\varepsilon} \lambda_{n}^{(N-2)\left(p-2^{*}\right) / 2}+C_{1} \varepsilon^{1 / 2^{*}} \quad \text { for all } n
$$

so that

$$
\limsup _{n \rightarrow \infty} a_{n} \leq C_{1} \varepsilon^{1 / 2^{*}}
$$

since $\lambda_{n} \rightarrow \infty$ and $(N-2)\left(p-2^{*}\right) / 2<0$. Therefore, letting $\varepsilon \rightarrow 0$, one obtains $\lim _{n \rightarrow \infty} a_{n}=0$, which contradicts 23 . Thus no diverging subsequence is allowed and the assertion is proved.

We are now able to easily conclude the proof of Theorem 4

Proof of Theorem 4 By the last Lemmata 24 and 25, up to a subsequence we can assume $\lambda_{n} \rightarrow \lambda \neq 0$. Thus, from $T\left(\lambda_{n}, 0\right) u_{n} \rightarrow \tilde{u} \neq 0$ in $X_{s}$ we deduce

$$
u_{n} \rightarrow u:=T(\lambda, 0)^{-1} \tilde{u} \neq 0 \quad \text { in } X_{s}
$$

(up to a subsequence) by Remark 12 Therefore, recalling from Lemma 21 that $I^{\prime}\left(u_{n}\right)$ $\rightarrow 0$ in $X_{s}^{\prime}$, by Proposition 14 one concludes that $u \in X_{s}$ is a critical point for $I_{\mid X_{s}}$. Hence $I^{\prime}(u)=0$ in $X^{\prime}$ (see Remark 16), i.e., $u$ satisfies $(8)$. Since the nonnegativity of $u$ easily follows from hypothesis $\left(\mathbf{f}_{2}\right)$, the proof is complete. 


\section{The extendibility result}

In this section we assume that $f \in C(\mathbb{R} ; \mathbb{R})$ satisfies condition $\left(\mathbf{f}_{1}^{\prime}\right)$ and $u \in X$ is such that $u \geq 0$ and

$$
\int_{\mathbb{R}^{N}} \nabla u \cdot \nabla \varphi d x+\int_{\mathbb{R}^{N}} \frac{u \varphi}{|y|^{2}} d x=\int_{\mathbb{R}^{N}} f(u) \varphi d x \quad \text { for all } \varphi \in X .
$$

Recall from Remark 7 that $X \supset H^{1}$ if $k>2$. We are going to show that 25 holds true for every $\varphi \in H^{1}$ also in the case $k=2$. Accordingly, in this section we write $x=(y, z) \in \mathbb{R}^{2} \times \mathbb{R}^{N-2}$ with $N>2$. As a result, this proves Proposition 5 and allows us to conclude that Theorem 4 actually provides a weak solution to equation $(6)$, in the sense of Definition 2 .

Our goal will be achieved in several steps.

Step 1. We begin by proving 25 for any $\varphi \in H^{1}\left(\mathbb{R}^{N}\right)$ satisfying $\varphi \geq 0, \varphi \in L^{\infty}\left(\mathbb{R}^{N}\right)$ and $\operatorname{supp} \varphi \subset B_{R}^{(2)} \times B_{R}^{(N-2)}$ for some $R>0$.

For this purpose, consider a sequence $\left\{\eta_{n}\right\} \subset C^{\infty}\left(\mathbb{R}^{N} ; \mathbb{R}\right)$ defined as follows: given $\eta \in C^{\infty}(\mathbb{R} ; \mathbb{R})$ such that $0 \leq \eta \leq 1, \eta=0$ in $(-\infty, 1]$ and $\eta=1$ in $[2, \infty)$, set $\eta_{n}(x):=\eta(n|y|)$ for any $n \in \overline{\mathbb{N}}$ such that $1 / n<R$. Thus, for all $n$ one has

- $0 \leq \eta_{n} \leq 1$,

- $\eta_{n}=0$ in $\bar{B}_{1 / n} \times \mathbb{R}^{N-2}$ and $\eta_{n}=1$ in $\left(\mathbb{R}^{2} \backslash B_{2 / n}\right) \times \mathbb{R}^{N-2}$,

- $\left|\nabla \eta_{n}\right| \leq n\left\|\eta^{\prime}\right\|_{L^{\infty}(\mathbb{R})}$ and $\left|\nabla \eta_{n}\right|=0$ in $\left(\bar{B}_{1 / n} \cup\left(\mathbb{R}^{2} \backslash B_{2 / n}\right)\right) \times \mathbb{R}^{N-2}$.

Moreover, $\eta_{n} \rightarrow 1$ almost everywhere in $\mathbb{R}^{N}$.

Since $\eta_{n} \in C^{\infty}$ and $\varphi \in H^{1}$, it is easy to see that $\varphi \eta_{n} \in H^{1} \subset D^{1,2}$ with weak derivatives $\nabla\left(\varphi \eta_{n}\right)=\eta_{n} \nabla \varphi+\varphi \nabla \eta_{n}$. Moreover,

$$
\int_{\mathbb{R}^{N}} \frac{\varphi^{2} \eta_{n}^{2}}{|y|^{2}} d x=\int_{1 / n<|y|<R} \frac{\varphi^{2} \eta_{n}^{2}}{|y|^{2}} d x \leq n^{2} \int_{B_{R}^{(2)} \times B_{R}^{(N-2)}} \varphi^{2} d x \leq C\|\varphi\|_{L^{2^{*}}}
$$

As a consequence, $\varphi \eta_{n} \in X$ and therefore, by 25,

$$
\int_{\mathbb{R}^{N}} \nabla u \cdot \nabla \varphi \eta_{n} d x+\int_{\mathbb{R}^{N}} \nabla u \cdot \nabla \eta_{n} \varphi d x+\int_{\mathbb{R}^{N}} \frac{u \varphi \eta_{n}}{|y|^{2}} d x=\int_{\mathbb{R}^{N}} f(u) \varphi \eta_{n} d x .
$$

By the Lebesgue dominated convergence theorem, one obtains

$$
\lim _{n \rightarrow \infty} \int_{\mathbb{R}^{N}} \nabla u \cdot \nabla \varphi \eta_{n} d x=\int_{\mathbb{R}^{N}} \nabla u \cdot \nabla \varphi d x
$$

and

$$
\lim _{n \rightarrow \infty} \int_{\mathbb{R}^{N}} f(u) \varphi \eta_{n} d x=\int_{\mathbb{R}^{N}} f(u) \varphi d x
$$


On the other hand, setting $A_{n}:=\left\{x \in B_{R}^{(2)} \times B_{R}^{(N-2)}: 1 / n<|y|<2 / n\right\}$, we have $\left|A_{n}\right|=C / n^{2}$ and

$$
\begin{aligned}
\left|\int_{\mathbb{R}^{N}} \nabla u \cdot \nabla \eta_{n} \varphi d x\right| & \leq\|\varphi\|_{L^{\infty}} \int_{A_{n}}|\nabla u|\left|\nabla \eta_{n}\right| d x \\
& \leq C\left(\int_{A_{n}}|\nabla u|^{2} d x\right)^{1 / 2}\left(\int_{A_{n}}\left|\nabla \eta_{n}\right|^{2} d x\right)^{1 / 2} \\
& \leq C\left(\int_{A_{n}}|\nabla u|^{2} d x\right)^{1 / 2} n\left|A_{n}\right|^{1 / 2}=C\left(\int_{A_{n}}|\nabla u|^{2} d x\right)^{1 / 2}
\end{aligned}
$$

Since $\left|A_{n}\right| \rightarrow 0$, this implies

$$
\lim _{n \rightarrow \infty} \int_{\mathbb{R}^{N}} \nabla u \cdot \nabla \eta_{n} \varphi d x=0 .
$$

We now use the assumption $u \geq 0$ to estimate the integrals $\int_{\mathbb{R}^{N}}|y|^{-2} u \varphi \eta_{n} d x$ as $n \rightarrow \infty$. From 26-29, we get

$$
\lim _{n \rightarrow \infty} \int_{\mathbb{R}^{N}} \frac{u \varphi \eta_{n}}{|y|^{2}} d x=\int_{\mathbb{R}^{N}} f(u) \varphi d x-\int_{\mathbb{R}^{N}} \nabla u \cdot \nabla \varphi d x
$$

and, since $0 \leq|y|^{-2} u \varphi \eta_{n} \rightarrow|y|^{-2} u \varphi$ almost everywhere in $\mathbb{R}^{N}$, by the Fatou lemma we deduce $|y|^{-2} u \varphi \in L^{1}\left(\mathbb{R}^{N}\right)$. Since $|y|^{-2} u \varphi \eta_{n} \leq|y|^{-2} u \varphi$, the Lebesgue dominated convergence theorem finally yields

$$
\lim _{n \rightarrow \infty} \int_{\mathbb{R}^{N}} \frac{u \varphi \eta_{n}}{|y|^{2}} d x=\int_{\mathbb{R}^{N}} \frac{u \varphi}{|y|^{2}} d x
$$

As a result, from $30-31$ we infer that 25 also holds for every $\varphi \in H^{1}$ such that $\varphi \geq 0, \varphi \in L^{\infty}$ and $\operatorname{supp} \varphi$ is bounded.

Step 2. Now consider $\varphi \in H^{1}\left(\mathbb{R}^{N}\right)$ with $\varphi \geq 0$ and $\varphi \in L^{\infty}\left(\mathbb{R}^{N}\right)$.

We will use a truncating sequence $\left\{\zeta_{n}\right\} \subset C_{c}^{\infty}\left(\mathbb{R}^{N} ; \mathbb{R}\right)$, defined as follows: given $\zeta \in C^{\infty}(\mathbb{R} ; \mathbb{R})$ such that $0 \leq \zeta \leq 1, \zeta=1$ in $(-\infty, 1]$ and $\zeta=0$ in $[2, \infty)$, set $\zeta_{n}(x):=\zeta\left(n^{-1}|x|\right)$ for any $n \in \mathbb{N} \backslash\{0\}$. Thus, for all $n$ one has

- $0 \leq \zeta_{n} \leq 1$,

- $\zeta_{n}=1$ in $\bar{B}_{n}$ and $\zeta_{n}=0$ in $\mathbb{R}^{N} \backslash B_{2 n}$,

- $\left|\nabla \zeta_{n}\right| \leq\left\|\zeta^{\prime}\right\|_{L^{\infty}(\mathbb{R})}$.

Moreover, $\zeta_{n} \rightarrow 1$ pointwise in $\mathbb{R}^{N}$.

Since $\zeta_{n} \in C_{c}^{\infty}$ and $\varphi \in H^{1}$, it is easy to see that $\varphi \zeta_{n} \in H^{1}$ with weak derivatives $\nabla\left(\varphi \zeta_{n}\right)=\zeta_{n} \nabla \varphi+\varphi \nabla \zeta_{n}$. Clearly $\varphi \zeta_{n} \in L^{\infty}\left(\mathbb{R}^{N}\right)$ is nonnegative and compactly supported. Hence the result of Step 1 yields

$$
\int_{\mathbb{R}^{N}} \nabla u \cdot \nabla \varphi \zeta_{n} d x+\int_{\mathbb{R}^{N}} \nabla u \cdot \nabla \zeta_{n} \varphi d x+\int_{\mathbb{R}^{N}} \frac{u \varphi \zeta_{n}}{|y|^{2}} d x=\int_{\mathbb{R}^{N}} f(u) \varphi \zeta_{n} d x
$$


and we argue as before to obtain 25 . Here the convergence

$$
\lim _{n \rightarrow \infty} \int_{\mathbb{R}^{N}} \nabla u \cdot \nabla \zeta_{n} \varphi d x=0
$$

plainly follows by the Lebesgue dominated convergence theorem.

Step 3. We now drop the boundedness requirement and consider $\varphi \in H^{1}\left(\mathbb{R}^{N}\right)$ such that $\varphi \geq 0$.

For all $n \in \mathbb{N}$, set $\varphi_{n}:=\min (n, \varphi)=\varphi-(\varphi-n)_{+}$. It is obvious that $0 \leq \varphi_{n} \leq n$ and it is easy to see that $\varphi_{n} \rightarrow \varphi$ in $H^{1}$. Hence, by Step 2 , we have

$$
\int_{\mathbb{R}^{N}} \nabla u \cdot \nabla \varphi_{n} d x+\int_{\mathbb{R}^{N}} \frac{u \varphi_{n}}{|y|^{2}} d x=\int_{\mathbb{R}^{N}} f(u) \varphi_{n} d x
$$

with $\lim _{n \rightarrow \infty} \int_{\mathbb{R}^{N}} \nabla u \cdot \nabla \varphi_{n} d x=\int_{\mathbb{R}^{N}} \nabla u \cdot \nabla \varphi d x$ and $\lim _{n \rightarrow \infty} \int_{\mathbb{R}^{N}} f(u) \varphi_{n} d x=$ $\int_{\mathbb{R}^{N}} f(u) \varphi d x$ (recall that $f(u) \in L^{2^{*} /\left(2^{*}-1\right)}$ and $\varphi_{n} \rightarrow \varphi$ in $L^{2^{*}}$ ). Since $\varphi_{n} \leq \varphi$, the same argument of Step 1 finally shows that

$$
\lim _{n \rightarrow \infty} \int_{\mathbb{R}^{N}} \frac{u \varphi_{n}}{|y|^{2}} d x=\int_{\mathbb{R}^{N}} \frac{u \varphi}{|y|^{2}} d x .
$$

Step 4. Without any assumption on the sign of $\varphi \in H^{1}\left(\mathbb{R}^{N}\right)$, one can apply the result of Step 3 to both $\varphi_{+}, \varphi_{-} \geq 0$ and then deduce (25) for $\varphi=\varphi_{+}-\varphi_{-}$.

\section{Properties of weak solutions}

In this section we study asymptotic and summability properties of nonnegative weak solutions of equation (6), which amounts to proving Proposition 6 and thus, by Theorem 4 and Proposition 5, to concluding the proof of Theorem 3 Accordingly, we assume that $f \in C(\mathbb{R} ; \mathbb{R})$ satisfies condition $\left(\mathbf{f}_{1}^{\prime}\right)$ and $u \geq 0$ is a weak solution in the sense of Definition 2

Let us recall the following result from [22].

Theorem 26 ([22]). Assume that $h \in D^{1,2}\left(\mathbb{R}^{N}\right)$ is nonnegative and satisfies

$$
\int_{\mathbb{R}^{N}} \nabla h \cdot \nabla \varphi d x \leq \int_{\mathbb{R}^{N}} \phi(x, h) \varphi d x \quad \text { for all } \varphi \in C_{c}^{\infty}\left(\mathbb{R}^{N}\right), \varphi \geq 0,
$$

where the measurable function $\phi: \mathbb{R}^{N} \times \mathbb{R} \rightarrow \mathbb{R}$ is such that

$$
0 \leq \phi(x, s) \leq b(x) s^{a} \quad \text { for all } s \geq 0 \text { and almost every } x \in \mathbb{R}^{N}
$$

with $1<a<2^{*}-1$ and $b \in L^{2^{*} /\left(2^{*}-1-a\right)}\left(\mathbb{R}^{N}\right)$. Then $h$ is bounded in a neighbourhood of the origin and $\lim \sup _{|x| \rightarrow \infty}|x|^{N-2} h(x)<\infty$. 
With a view to applying Theorem 26, fix $a \in\left(1,2^{*}-1\right)$ and define

$$
\phi(x, s):=\left|f\left(u(x)^{\left(2^{*}-1-a\right) /\left(2^{*}-1\right)}|s|^{a /\left(2^{*}-1\right)}\right)\right| \quad \text { for all } x \in \mathbb{R}^{N} \text { and } s \in \mathbb{R} .
$$

Since $\phi(x, u(x))=|f(u(x))|$ and $u$ is a nonnegative weak solution, one has

$$
\begin{aligned}
\int_{\mathbb{R}^{N}} \nabla u \cdot \nabla \varphi d x & \leq \int_{\mathbb{R}^{N}} \nabla u \cdot \nabla \varphi d x+\int_{\mathbb{R}^{N}} \frac{u \varphi}{|y|^{2}} d x=\int_{\mathbb{R}^{N}} f(u) \varphi d x \\
& \leq \int_{\mathbb{R}^{N}} \phi(x, u) \varphi d x
\end{aligned}
$$

for all $\varphi \in C_{c}^{\infty}\left(\mathbb{R}^{N}\right), \varphi \geq 0$. Moreover

$$
\phi(x, s) \leq M\left|u(x)^{\left(2^{*}-1-a\right) /\left(2^{*}-1\right)} s^{a /\left(2^{*}-1\right)}\right|^{2^{*}-1}=M u(x)^{2^{*}-1-a} s^{a}
$$

by assumption $\left(\mathbf{f}_{1}^{\prime}\right)$. Since $u^{2^{*}-1-a} \in L^{2^{*} /\left(2^{*}-1-a\right)}\left(\mathbb{R}^{N}\right)$, by Theorem 26 we conclude that $u$ is bounded in a neighbourhood of the origin and satisfies

$$
\limsup _{|x| \rightarrow \infty}|x|^{N-2} u(x)<\infty
$$

Now we set $\widetilde{z}:=(0, z)$ for any $z \in \mathbb{R}^{N-k}$ and observe that $u(\cdot-\widetilde{z}) \geq 0$ is still a weak solution to equation (6). Hence, by repeating the above argument, one infers that for every $z \in \mathbb{R}^{N-k}$ there exist $\rho_{z}, C_{z}>0$ such that $u(\cdot-\widetilde{z}) \leq C_{z}$ almost everywhere in $B_{\rho_{z}}^{(N)}$, i.e., $u \leq C_{z}$ almost everywhere in $B_{\rho_{z}}^{(N)}(\widetilde{z})$. On the other hand $u$ is of class $C^{2}$ on $\mathbb{R}^{N} \backslash\left(\{0\} \times \mathbb{R}^{N-k}\right)$, by standard elliptic regularity theory. These facts, together with 32 , imply $u \in L^{\infty}\left(\mathbb{R}^{N}\right)$.

In order to show that $u \in L^{2}\left(\mathbb{R}^{N}\right)$, we are now going to improve the asymptotic estimate (32). Let us begin with some preliminaries. First, for $\varepsilon \in(0,1)$ define

$$
\alpha_{\varepsilon}:=\frac{N-2+\sqrt{(N-2)^{2}+4(1-\varepsilon)}}{2} .
$$

Notice that $\alpha_{\varepsilon}$ satisfies the equation $\alpha_{\varepsilon}^{2}-(N-2) \alpha_{\varepsilon}-(1-\varepsilon)=0$. Moreover, we can assume $2 \alpha_{\varepsilon}>N$ by taking $\varepsilon$ small enough. Secondly, by means of 32 and assumption $\left(\mathbf{f}_{1}^{\prime}\right)$, let $C_{1}>0$ and $R_{\infty}>0$ be such that

$$
f(u) \leq M u^{2^{*}-2} u \leq C_{1} \frac{u}{|x|^{4}} \quad \text { for almost every }|x| \geq R_{\infty} .
$$

Then take $R \geq R_{\infty}$ such that $|x| \geq R \Rightarrow C_{1}|x|^{-2} \leq \varepsilon$ and set $\Omega:=\mathbb{R}^{N} \backslash \bar{B}_{R}$.

By definition of weak solution, we have

$$
\begin{aligned}
\int_{\Omega} \nabla u \cdot \nabla \varphi d x & =\int_{\Omega} f(u) \varphi d x-\int_{\Omega} \frac{u \varphi}{|y|^{2}} d x \leq \int_{\Omega} C_{1} \frac{u \varphi}{|x|^{4}} d x-\int_{\Omega} \frac{u \varphi}{|x|^{2}} d x \\
& \leq-(1-\varepsilon) \int_{\Omega} \frac{u \varphi}{|x|^{2}} d x
\end{aligned}
$$

for all $\varphi \in X$ such that $\varphi \geq 0$ and $\operatorname{supp} \varphi \subset \Omega$. 
On the other hand, the function defined by $v(x):=|x|^{-\alpha_{\varepsilon}}$ is such that $|\nabla v|=$ $\alpha_{\varepsilon}|x|^{-\alpha_{\varepsilon}-1}$ and $\Delta v=\left(\alpha_{\varepsilon}^{2}-(N-2) \alpha_{\varepsilon}\right)|x|^{-2} v=(1-\varepsilon)|x|^{-2} v$. Thus $v \in H^{1}(\Omega)$ and

$$
\int_{\Omega} \nabla v \cdot \nabla \varphi d x=-(1-\varepsilon) \int_{\Omega} \frac{v \varphi}{|x|^{2}} d x
$$

for all $\varphi \in C_{c}^{\infty}(\Omega)$. Since $2 \alpha_{\varepsilon}>N$ implies $|x|^{-2} v \in L^{2^{*} /\left(2^{*}-1\right)}(\Omega)$, a density argument shows that 34 also holds true for all $\varphi \in D_{0}^{1,2}(\Omega)$.

Now, take a constant $C_{2}=C_{2}(u, \varepsilon, R)>0$ such that $C_{2} v \geq u$ almost everywhere on the open annulus

$$
A:=\left\{x \in \mathbb{R}^{N}: R<|x|<R+1\right\} \subset \Omega
$$

and introduce the functions

$$
w:=C_{2} v-u \quad \text { and } \quad \bar{w}:=\chi_{\Omega} w_{-}
$$

defined almost everywhere in $\mathbb{R}^{N}$. From

$$
0 \leq w_{-}=-\chi_{\{w<0\}} w=\chi_{\{w<0\}}\left(u-C_{2} v\right) \leq \chi_{\{w<0\}} u \in L^{2^{*}}\left(\mathbb{R}^{N}\right)
$$

we derive that $\bar{w} \geq 0$ satisfies $\bar{w} \in L^{2^{*}}\left(\mathbb{R}^{N}\right)$ and $|y|^{-2} \bar{w}^{2} \in L^{1}\left(\mathbb{R}^{N}\right)$. Taking into account that $w \in D^{1,2}(\Omega)$ and $w_{-}=0$ almost everywhere in $A$, so that $\nabla w_{-}=0$ almost everywhere in $A$, it is a standard exercise to check that $\bar{w} \in D_{0}^{1,2}(\Omega)$. Hence $\bar{w} \in X$ and we can use $\bar{w}$ as a test function in both (33) and (34). So, upon multiplying (34) by $C_{2}$ and subtracting [33], we obtain

$$
\int_{\Omega} \nabla w \cdot \nabla w_{-} d x \geq-(1-\varepsilon) \int_{\Omega} \frac{w w_{-}}{|x|^{2}} d x,
$$

that is,

$$
-\int_{\Omega}\left|\nabla w_{-}\right|^{2} d x \geq(1-\varepsilon) \int_{\Omega} \frac{w_{-}^{2}}{|x|^{2}} d x .
$$

This implies

$$
\|\bar{w}\|_{D_{0}^{1,2}(\Omega)}=\left\|\nabla w_{-}\right\|_{L^{2}(\Omega)}=0,
$$

which means $u \leq C_{2} v \in L^{2}(\Omega)$ almost everywhere in $\Omega$. Therefore $u \in L^{2}\left(\mathbb{R}^{N}\right)$ and

$$
\limsup _{|x| \rightarrow \infty}|x|^{v} u(x)<\infty
$$

for any $v \leq \alpha_{\varepsilon}$. Since $\alpha_{\varepsilon}$ decreases as $\varepsilon$ increases, we conclude that (35) holds true for every $v<\lim _{\varepsilon \rightarrow 0} \alpha_{\varepsilon}=\left(N-2+\sqrt{(N-2)^{2}+4}\right) / 2$. 


\section{Applications to nonlinear field equations}

In this section we take $N=3$ and apply the result of Theorem 3 to the problem of finding solitary waves for nonlinear evolution equations of the Schrödinger or Klein-Gordon type. Roughly speaking, a solitary wave is a finite-energy solution of a field equation whose energy density travels as a localized packet and, owing to this particle-like behaviour, it can be regarded as an extended particle, in contrast to point particles. In addition, the solitary waves preserve intrinsic properties of particles such as the angular momentum. For an introduction to solitary solutions to evolution equations the reader is referred to [3], [7]. In this paper we are interested in the existence of solitary waves with nonvanishing angular momentum. Consider for instance the nonlinear Schrödinger equation

$$
i \frac{\partial \psi}{\partial t}=-\Delta \psi+\omega \psi-f(|\psi|)|\psi|^{-1} \psi, \quad(x, t) \in \mathbb{R}^{3} \times \mathbb{R},
$$

where $\psi(x, t)=\psi_{1}(x, t)+i \psi_{2}(x, t) \in \mathbb{C}, \omega$ is a real constant and $f \in C(\mathbb{R} ; \mathbb{R})$ satisfies $f(0)=0$. By the Noether theorem, any invariance of equation (36) under the action of a group of transformations smoothly depending on one parameter yields a conservation law; this means that any solution $\psi$ having a suitable spatial decay bears a constant of motion, which represents a relevant physical quantity. In particular, time translation invariance yields the conservation of the energy, which is given by

$$
\begin{aligned}
E(\psi) & =\frac{1}{2} \int_{\mathbb{R}^{3}}\left[|\nabla \psi|^{2}+\omega|\psi|^{2}\right] d x-\int_{\mathbb{R}^{3}} F(|\psi|) d x \\
& =\frac{1}{2} \int_{\mathbb{R}^{3}}\left[|\nabla u|^{2}+|\nabla S|^{2} u^{2}+\omega u^{2}\right] d x-\int_{\mathbb{R}^{3}} F(u) d x
\end{aligned}
$$

where $F(t)=\int_{0}^{t} f(s) d s$ and we have used the polar form

$$
\psi(x, t)=u(x, t) e^{i S(x, t)}, \quad u(x, t) \geq 0, \quad S(x, t) \in \mathbb{R} .
$$

As equation (36) is invariant under space rotations, also the angular momentum

$$
\mathbf{M}(\psi)=\operatorname{Re} \int_{\mathbb{R}^{3}} i \bar{\psi} x \wedge \nabla \psi d x=-\int_{\mathbb{R}^{3}} u^{2} x \wedge \nabla S d x
$$

is constant in time. Observe that $\mathbf{M}=0$ whenever $S$ does not depend on $x$. In order to prevent the vanishing of $\mathbf{M}$, we look for standing solutions of the form

$$
\psi(x, t)=\varphi(x) e^{-i \Omega t}, \quad \varphi(x) \in \mathbb{C}, \quad \Omega \in \mathbb{R} .
$$

In this case equation (36) reduces to

$$
-\Delta \varphi+(\omega-\Omega) \varphi=f(|\varphi|)|\varphi|^{-1} \varphi \quad \text { in } \mathbb{R}^{3} .
$$

If we now make the ansatz

$$
\varphi(x)=u(x) e^{i \vartheta(x)}, \quad u(x) \geq 0, \quad \vartheta(x) \in \mathbb{R} / 2 \pi \mathbb{Z},
$$


then equation (40) (and so equation (36) ) is equivalent to the system

$$
\left\{\begin{array}{l}
-\Delta u+(\omega-\Omega) u+|\nabla \vartheta|^{2} u=f(u) \\
2 \nabla u \cdot \nabla \vartheta+u \Delta \vartheta=0 .
\end{array}\right.
$$

This suggests choosing as phase function the smooth map $\vartheta: \mathbb{R}^{2} \backslash\{0\} \rightarrow \mathbb{R} / 2 \pi \mathbb{Z}$ (constant in $z$ ) which gives the angle of the cylindrical coordinates in $\mathbb{R}^{3}$, that is,

$$
\vartheta(y):= \begin{cases}\arctan \left(y_{2} / y_{1}\right) & \text { if } y_{1}>0, \\ \arctan \left(y_{2} / y_{1}\right)+\pi & \text { if } y_{1}<0, \\ \pi / 2 & \text { if } y_{1}=0 \text { and } y_{2}>0, \\ -\pi / 2 & \text { if } y_{1}=0 \text { and } y_{2}<0\end{cases}
$$

(up to composition with the projection $\mathbb{R} \rightarrow \mathbb{R} / 2 \pi \mathbb{Z}$ ). Hence we have

$$
\Delta \vartheta=0, \quad \nabla \vartheta \cdot y=0, \quad|\nabla \vartheta|^{2}=\frac{1}{|y|^{2}}
$$

so that, if $u(y, z)=u(|y|, z)$, system (41) reduces to its first equation, which becomes

$$
-\Delta u+(\omega-\Omega) u+\frac{1}{|y|^{2}} u=f(u) \quad \text { in } \mathbb{R}^{3} .
$$

Using $H^{1}$ variational techniques, it is quite standard to see that this equation admits nonnegative cylindrical solutions when $\Omega<\omega$. Here we are concerned with the limit case $\Omega=\omega$ and, by Theorem 3 , we can prove the following existence results.

Theorem 27. Assume that $f \in C(\mathbb{R} ; \mathbb{R})$ satisfies hypotheses $\left(\mathbf{f}_{1}\right)-\left(\mathbf{f}_{4}\right)$. Let $u \in H^{1}\left(\mathbb{R}^{3}\right)$ be the solution found in Theorem 3 and $\vartheta \in C^{\infty}\left(\mathbb{R}^{2} \backslash\{0\} ; \mathbb{R} / 2 \pi \mathbb{Z}\right)$ be given by 42 . Then $\varphi(x):=u(x) e^{i \vartheta(y)}$ defines a nontrivial classical solution to the complex equation

$$
-\Delta \varphi=f(|\varphi|)|\varphi|^{-1} \varphi \quad \text { in } \mathbb{R}^{3}
$$

satisfying $\varphi \in H^{1} \cap L^{\infty}\left(\mathbb{R}^{3}\right)$ and $\varphi(x)=O\left(|x|^{-\nu}\right)$ as $|x| \rightarrow \infty$ for every $v<$ $(1+\sqrt{5}) / 2$.

Theorem 28. Assume that $f \in C(\mathbb{R} ; \mathbb{R})$ satisfies hypotheses $\left(\mathbf{f}_{1}\right)-\left(\mathbf{f}_{4}\right)$. Let $u \in H^{1}\left(\mathbb{R}^{3}\right)$ be the solution found in Theorem 3 and $\vartheta \in C^{\infty}\left(\mathbb{R}^{2} \backslash\{0\} ; \mathbb{R} / 2 \pi \mathbb{Z}\right)$ be given by $(42$. Then $\psi(x, t):=u(x) e^{i[\vartheta(y)-\omega t]}$ defines a nontrivial classical solution to equation 36 such that

(i) $\psi(x, t)=O\left(|x|^{-v}\right)$ as $|x| \rightarrow \infty$ (uniformly in $t \in \mathbb{R}$ ) for any $v<(1+\sqrt{5}) / 2$;

(ii) $E(\psi)=I(u)+\frac{1}{2} \omega\|u\|_{L^{2}\left(\mathbb{R}^{3}\right)}^{2}<\infty$;

(iii) $\mathbf{M}(\psi)=-\left(0,0,\|u\|_{L^{2}\left(\mathbb{R}^{3}\right)}^{2}\right) \neq 0$. 
Remark 29. Theorem 28 also gives the existence of travelling solitary waves with nonvanishing angular momentum. Indeed, a solution $\psi_{\mathbf{v}}$ travelling with any vector velocity $\mathbf{v}$ can be obtained from a standing solution $\psi=\varphi(x) e^{-i \omega t}$ by setting

$$
\psi_{\mathbf{v}}(x, t)=\varphi(x-t \mathbf{v}) e^{i\left[\frac{1}{2} \mathbf{v} \cdot x-\left(\frac{1}{4}|\mathbf{v}|^{2}+\omega\right) t\right]} .
$$

In particular, if $\psi$ is the solution given by Theorem 28 and $\mathbf{v}=(0,0, v)$, then by an obvious change of variable it is easy to see that $E\left(\psi_{\mathbf{v}}\right)=E(\psi)+\frac{v^{2}}{8}\|u\|_{L^{2}\left(\mathbb{R}^{3}\right)}^{2}$. Moreover, one obtains again $\mathbf{M}\left(\psi_{\mathbf{v}}\right)=-\left(0,0,\|u\|_{L^{2}\left(\mathbb{R}^{3}\right)}^{2}\right)$. Note that also $\psi_{\mathbf{v}}$ is well localized in space for all $t \in \mathbb{R}$.

Remark 30. The same arguments yield the existence of standing and travelling solitary waves with nonvanishing angular momentum for nonlinear Klein-Gordon equations

$$
\frac{\partial^{2} \psi}{\partial t^{2}}-\Delta \psi+\omega^{2} \psi-f(|\psi|)|\psi|^{-1} \psi=0, \quad(x, t) \in \mathbb{R}^{3} \times \mathbb{R} .
$$

See [3] for a discussion of such equations. On physical grounds, the solitary waves of 44] exhibit all the most characteristic features of relativistic particles (under the assumption that the speed of light equals 1). The conserved energy and angular momentum of a wave solution (38) are given by

$$
\begin{aligned}
E(\psi) & =\frac{1}{2} \int_{\mathbb{R}^{3}}\left[\left|\frac{\partial \psi}{\partial t}\right|^{2}+|\nabla \psi|^{2}+\omega^{2}|\psi|^{2}\right] d x-\int_{\mathbb{R}^{3}} F(|\psi|) d x \\
& =\frac{1}{2} \int_{\mathbb{R}^{3}}\left[|\nabla u|^{2}+\left(|\nabla S|^{2}+\left(\frac{\partial S}{\partial t}\right)^{2}+\omega^{2}\right) u^{2}+\left(\frac{\partial u}{\partial t}\right)^{2}\right] d x-\int_{\mathbb{R}^{3}} F(u) d x
\end{aligned}
$$

and

$$
\mathbf{M}(\psi)=\operatorname{Re} \int_{\mathbb{R}^{3}} \frac{\overline{\partial \psi}}{\partial t} x \wedge \nabla \psi d x=\int_{\mathbb{R}^{3}} x \wedge\left(\frac{\partial u}{\partial t} \nabla u+u^{2} \frac{\partial S}{\partial t} \nabla S\right) d x .
$$

Ansatz $\psi=\varphi(x) e^{-i \omega t}$ in 44 leads again to equation 43. Then, by Lorentz invariance, a travelling solution $\psi_{\mathbf{v}}$ is obtained by boosting; for example if $\mathbf{v}=(v, 0,0), v<1$, then $\psi_{\mathbf{v}}(x, t)=\varphi\left(\gamma\left[y_{1}-v t\right], y_{2}, z\right) e^{i \gamma \omega\left(v y_{1}-t\right)}$ where $\gamma=\left(1-v^{2}\right)^{-1 / 2}$.

Proof of Theorem 27 For all $x=(y, z) \in \mathcal{O}:=\left(\mathbb{R}^{2} \backslash\{0\}\right) \times \mathbb{R}$, we set

$$
\varphi(x):=u(x) e^{i \vartheta(y)} .
$$

Clearly $\varphi \in L^{\infty} \cap L^{2}\left(\mathbb{R}^{3}\right)$ and $\lim \sup _{|x| \rightarrow \infty}|x|^{v}|\varphi(x)|=0$ for every $v<(1+\sqrt{5}) / 2$ (Theorem 3). Moreover from $\nabla \varphi=e^{i \vartheta}(\nabla u+i u \nabla \vartheta)$ one derives $|\nabla \varphi|^{2}=|\nabla u|^{2}+$ $|y|^{-2} u^{2} \in L^{1}\left(\mathbb{R}^{3}\right)$, so that $\varphi \in H^{1}\left(\mathbb{R}^{3}\right)$. We now prove that $\varphi$ is a distributional solution to equation 43 on the open set $\mathcal{O}$, that is,

$$
\int_{\mathcal{O}} \nabla \varphi \cdot \nabla \xi d x=\int_{\mathcal{O}} f(|\varphi|)|\varphi|^{-1} \varphi \xi d x \quad \text { for all } \xi \in C_{c}^{\infty}(\mathcal{O} ; \mathbb{C}),
$$


or equivalently,

$$
\int_{\mathcal{O}} \nabla \varphi \cdot \nabla\left(e^{-i \vartheta} \xi\right) d x=\int_{\mathcal{O}} f(|\varphi|)|\varphi|^{-1} \varphi e^{-i \vartheta} \xi d x \quad \text { for all } \xi \in C_{c}^{\infty}(\mathcal{O} ; \mathbb{C}) .
$$

With a slight abuse of notation, we write $h \cdot k=h_{1} k_{1}+h_{2} k_{2}+h_{3} k_{3}$ also for $h, k \in \mathbb{C}^{3}$. On the one hand, we have

$$
\int_{\mathcal{O}} f(|\varphi|)|\varphi|^{-1} \varphi e^{-i \vartheta} \xi d x=\int_{\mathcal{O}} f(u) \xi d x=\int_{\mathbb{R}^{3}} f(u) \alpha d x+i \int_{\mathbb{R}^{3}} f(u) \beta d x
$$

where $\xi=\alpha+i \beta$ with $\alpha, \beta \in C_{c}^{\infty}(\mathcal{O} ; \mathbb{R})$. On the other hand,

$$
\begin{aligned}
\int_{\mathcal{O}} \nabla \varphi \cdot \nabla\left(e^{-i \vartheta} \xi\right) d x & =\int_{\mathcal{O}}\left(e^{i \vartheta} \nabla u+i u e^{i \vartheta} \nabla \vartheta\right) \cdot\left(e^{-i \vartheta} \nabla \xi-i \xi e^{-i \vartheta} \nabla \vartheta\right) d x \\
& =\int_{\mathcal{O}}(\nabla u \cdot \nabla \xi+u \xi \nabla \vartheta \cdot \nabla \vartheta) d x+i \int_{\mathcal{O}} u \nabla \vartheta \cdot \nabla \xi d x \\
& =\int_{\mathcal{O}}\left(\nabla u \cdot \nabla \xi+u \xi|\nabla \vartheta|^{2}\right) d x-i \int_{\mathcal{O}} \operatorname{div}(u \nabla \vartheta) \xi d x \\
& =\int_{\mathcal{O}}\left(\nabla u \cdot \nabla \xi+u \xi|y|^{-2}\right) d x \\
& =\int_{\mathbb{R}^{3}}\left(\nabla u \cdot \nabla \alpha+\frac{u \alpha}{|y|^{2}}\right) d x+i \int_{\mathbb{R}^{3}}\left(\nabla u \cdot \nabla \beta+\frac{u \beta}{|y|^{2}}\right) d x
\end{aligned}
$$

where we have taken into account that $\nabla u \cdot \nabla \vartheta=0$ and $\operatorname{div}(u \nabla \vartheta)=\nabla u \cdot \nabla \vartheta+u \Delta \vartheta$ $=0$. Hence, observing that $C_{c}^{\infty}(\mathcal{O} ; \mathbb{R}) \subset X\left(\mathbb{R}^{3}\right)$, we conclude by Definition 2 of weak solution. By the same extendibility argument used in the proof of Proposition 5 (Section 5, one can show that $\varphi$ is a distributional solution to (43) even on the whole space $\mathbb{R}^{3}$. Finally, standard regularity arguments (see for example [25]) show that $\varphi$ is actually a classical solution to 43 on $\mathbb{R}^{3}$.

Proof of Theorem 28 For all $x=(y, z) \in\left(\mathbb{R}^{2} \backslash\{0\}\right) \times \mathbb{R}$ and $t \in \mathbb{R}$, we set

$$
\psi(x, t):=u(x) e^{i[\vartheta(y)-\omega t]} .
$$

Since $\varphi(x)=u(x) e^{i \vartheta(y)}$ defines a classical solution to 43 by Theorem 27 a straightforward substitution proves that $\psi$ is actually a classical solution to 36 on $\mathbb{R}^{3} \times \mathbb{R}$. Moreover $|\psi(x, t)|=|\varphi(x)|$ for all $(x, t) \in \mathbb{R}^{3} \times \mathbb{R}$ implies (i). In order to compute (ii) and (iii), notice that $\nabla \vartheta=|y|^{-2}\left(-y_{2}, y_{1}\right)$ and $x \wedge \nabla \vartheta=\left(-|y|^{-2} z y_{1},-|y|^{-2} z y_{2}, 1\right)$. Thus, by (37) the energy of $\psi$ turns out to be

$$
E(\psi)=\frac{1}{2} \int_{\mathbb{R}^{3}}\left[|\nabla u|^{2}+\frac{u^{2}}{|y|^{2}}+\omega u^{2}\right] d x-\int_{\mathbb{R}^{3}} F(u) d x=I(u)+\frac{\omega}{2} \int_{\mathbb{R}^{3}} u^{2} d x<\infty,
$$

whereas from 39 we deduce that the angular momentum of $\psi$ has the form

$$
\mathbf{M}(\psi)=\left(\int_{\mathbb{R}^{3}} z y_{1} \frac{u^{2}}{|y|^{2}} d x, \int_{\mathbb{R}^{3}} z y_{2} \frac{u^{2}}{|y|^{2}} d x,-\int_{\mathbb{R}^{3}} u^{2} d x\right) .
$$


Since $u \in L^{2}$ is nontrivial, one has $0 \neq \int_{\mathbb{R}^{3}} u^{2} d x<\infty$. On the other hand, it is easy to see that the first components of $\mathbf{M}(\psi)$ are vanishing. Indeed, by means of planar polar coordinates $\left(y_{1}, y_{2}\right)=(r \cos \phi, r \sin \phi)$, one obtains for instance

$$
\int_{\mathbb{R}^{3}} z y_{1} \frac{u^{2}}{|y|^{2}} d x=\int_{\mathbb{R}} z d z \int_{0}^{\infty} u^{2}(r, z) d r \int_{0}^{2 \pi} \cos \phi d \phi=0
$$

where the cylindrical symmetry $u(y, z)=u(r, z)$ has been used.

Acknowledgments. The authors wish to thank the referee for his meaningful advice.

\section{References}

[1] Ambrosetti, A., Felli, V., Malchiodi, A.: Ground states of nonlinear Schrödinger equations with potentials vanishing at infinity. J. Eur. Math. Soc. 7, 117-144 (2005) Zbl 1064.35175 MR 2120993

[2] Ambrosetti, A., Rabinowitz, P. H.: Dual variational methods in critical point theory and applications. J. Funct. Anal. 14, 349-381 (1973) Zbl 0273.49063 MR 0370183

[3] Badiale, M., Benci, V., Rolando, S.: Solitary waves: physical aspects and mathematical results. Rend. Sem. Mat. Univ. Pol. Torino 62, 107-154 (2004) Zbl pre05121560 MR 2131956

[4] Badiale, M., Rolando, S.: A note on nonlinear elliptic problems with singular potentials. Rend. Lincei Mat. Appl. 17, 1-16 (2006) Zbl pre05058596

[5] Badiale, M., Rolando, S.: Elliptic problems with singular potential and double-power nonlinearity. Mediterr. J. Math. 2, 417-436 (2005) Zbl pre05058641 MR 2192523

[6] Badiale, M., Tarantello, G.: A Sobolev-Hardy inequality with applications to a nonlinear elliptic equation arising in astrophysics. Arch. Ration. Mech. Anal. 163, 259-293 (2002) Zbl 1010.35041 MR 1918928

[7] Benci, V.: Solitons and Bohmian mechanics. Discrete Contin. Dyn. Syst. 8, 303-317 (2002) Zbl 0994.35102 MR 1897685

[8] Benci, V., D'Aprile, T.: The semiclassical limit of the nonlinear Schrödinger equation in a radial potential. J. Differential Equations 184, 109-138 (2002) Zbl 1060.35129 MR 1929149

[9] Benci, V., Fortunato, D.: A strongly degenerate elliptic equation arising from the semilinear Maxwell equations. C. R. Math. Acad. Sci. Paris 339, 839-842 (2004) Zbl pre02137982 MR 2111719

[10] Benci, V., Fortunato, D.: Towards a unified field theory for classical electrodynamics. Arch. Ration. Mech. Anal. 173, 379-414 (2004) Zbl 1065.78004 MR 2091510

[11] Benci, V., Grisanti, C. R., Micheletti, A.M.: Existence and nonexistence of the ground state solution for the nonlinear Schrödinger equations with $V(\infty)=0$. Topol. Methods Nonlinear Anal. 26, 203-219 (2005) Zbl 1105.35112 MR 2197759

[12] Benci, V., Micheletti, A. M.: Solutions in exterior domains of null mass nonlinear field equations. Adv. Nonlinear Stud. 6, 171-198 (2006) Zbl pre05039911 MR 2219834

[13] Benci, V., Visciglia, N.: Solitary waves with non-vanishing angular momentum. Adv. Nonlinear Stud. 3, 151-160 (2003) Zbl 1030.35051 MR 1955598

[14] Berestycki, H., Lions, P.-L.: Nonlinear scalar field equations. I. Existence of a ground state. Arch. Ration. Mech. Anal. 82, 313-345 (1983) Zbl 0533.35029 MR 0695535

[15] Bergh, J., Löfström, J.: Interpolation Spaces. Springer, Berlin (1976). Zbl 0344.46071 MR 0482275 
[16] Brezis, H., Dupaigne, L., Tesei, A.: On a semilinear elliptic equation with inverse-square potential. Selecta Math. (N.S.) 11, 1-7 (2005) Zbl pre05024139 MR 2179651

[17] Brezis, H., Vazquez, J. L.: Blow-up solutions of some nonlinear elliptic problems. Rev. Math. Univ. Complut. Madrid 10, 443-469 (1997) Zbl 0894.35038 MR 1605678

[18] Chabrowski, J.: On the nonlinear Neumann problem involving the critical Sobolev exponent and Hardy potential. Rev. Mat. Complut. 17, 195-227 (2004) Zbl pre02113563 MR 2063949

[19] D'Aprile, T.: On a class of solutions with non-vanishing angular momentum for nonlinear Schrödinger equations. Differential Integral Equations 16, 349-384 (2003) Zbl 1031.35130 MR 1947957

[20] Dupaigne, L.: A nonlinear elliptic PDE with the inverse-square potential. J. Anal. Math. 86, 359-398 (2002) Zbl 1034.35043 MR 1894489

[21] Dupaigne, L., Nedev, G.: Semilinear elliptic PDE's with a singular potential. Adv. Differential Equations 7, 973-1002 (2002) Zbl pre01977268 MR 1895114

[22] Egnell, H.: Asymptotic results for finite energy solutions of semilinear elliptic equations. J. Differential Equations 98, 34-56 (1992) Zbl 0778.35009 MR 1168970

[23] Felli, V., Terracini, S.: Elliptic equations with multi-singular inverse-square potentials and critical nonlinearity. Comm. Partial Differential Equations 31, 469-495 (2006) Zbl pre05025025 MR 2209764

[24] Ferrero, A., Gazzola, F.: Existence of solutions for singular critical growth semilinear elliptic equations. J. Differential Equations 177, 494-522 (2001) Zbl 0997.35017 MR 1876652

[25] Giaquinta, M.: Introduction to Regularity Theory for Nonlinear Elliptic Systems. Birkhäuser, Basel (1993) Zbl 0786.35001 MR 1239172

[26] Jabri, Y.: The Mountain Pass Theorem. Cambridge Univ. Press, Cambridge (2003) Zbl 1036.49001 MR 2012778

[27] Jannelli, E.: The role played by space dimension in elliptic critical problems. J. Differential Equations 156, 407-426 (1999) Zbl 0938.35058 MR 1705383

[28] Kavian, O.: Introduction à la théorie des points critiques et applications aux problèmes elliptiques. Springer, Paris (1993) Zbl 0797.58005 MR 1276944

[29] Pisani, L.: Remarks on the sum of Lebesgue spaces. Work in progress

[30] Rădulescu, V., Smets, D., Willem, M.: Hardy-Sobolev inequalities with remainder terms. Topol. Methods Nonlinear Anal. 20, 145-149 (2002) Zbl 1033.35044 MR 1940535

[31] Rolando, S.: Nonlinear elliptic equations with singular symmetric potentials. PhD Thesis, Dipartimento di Matematica, Univ. di Torino

[32] Ruiz, D., Willem, M.: Elliptic problems with critical exponents and Hardy potentials. J. Differential Equations 190, 524-538 (2003) Zbl pre01929521 MR 1970040

[33] Solimini, S.: A note on compactness-type properties with respect to Lorentz norms of bounded subsets of a Sobolev space. Ann. Inst. H. Poincaré Anal. Non Linéaire 12, 319-337 (1995) Zbl 0837.46025 MR 1340267

[34] Willem, M.: Minimax Theorems. Progr. Nonlinear Differential Equations 24, Birkhäuser, Boston (1996) Zbl 0856.49001 MR 1400007 